\title{
1 Dorsal striatum coding for the timely execution of action sequences
}

2 María Cecilia Martinez ${ }^{1,2}$, Camila Lidia Zold ${ }^{1}$, Mario Gustavo Murer ${ }^{1 *}$, Mariano Andrés Belluscio ${ }^{1 *}$.

3 1- Universidad de Buenos Aires y Consejo Nacional de Investigaciones Científicas y Técnicas

4 (CONICET), Instituto de Fisiología y Biofísica “Bernardo Houssay” (IFIBIO-Houssay), Grupo de

5 Neurociencia de Sistemas, Buenos Aires C1121ABG, Argentina.

6 2- Departamento de Fisiología, Biología Molecular y Celular “Dr. Héctor Maldonado”, Facultad de

7 Ciencias Exactas y Naturales, Universidad de Buenos Aires, CONICET, Ciudad Universitaria Pabellón 2, 8 AR1428EHA Buenos Aires, Argentina.

9 Author list footnotes*. Co-senior authors

10 *Correspondence: mbellu@fmed.uba.ar, gmurer@fmed.uba.ar .

\section{Summary}

12 The automatic initiation of actions can be highly functional. But occasionally these actions cannot

13 be withheld and are released at inappropriate times, impulsively. Striatal activity has been shown to

14 participate in the timing of action sequence initiation and it has been linked to impulsivity. Using a self-

15 initiated task, we trained adult rats to withhold a rewarded action sequence until a waiting time interval

16 has elapsed. By analyzing neuronal activity we show that the striatal response preceding the initiation of

17 the learned sequence is strongly modulated by the time subjects wait before eliciting the sequence.

18 Interestingly, the modulation is steeper in adolescent rats, which show a strong prevalence of impulsive

19 responses compared to adults. We hypothesize this anticipatory striatal activity reflects the animals' 
20 subjective reward expectation, based on the elapsed waiting time, while its steeper waiting modulation in

21 adolescence reflects age-related differences in temporal discounting, internal urgency states or explore22 exploit balance.

23 Keywords: Dorsal Striatum, Rat, Reward, Action sequence, Waiting time, Impulsivity, Adolescence, 24 Reinforcement learning.

\section{Introduction}

The striatum is involved in the acquisition and execution of action sequences (Costa, 2011;

27 Graybiel, 1998; Hikosaka et al., 1999). It has also been linked to temporal information processing 28 (Bakhurin et al., 2017; Emmons et al., 2017; Gouvêa et al., 2015; Matell et al., 2003; Mello et al., 2015)

29 and to the use of temporal information for the action initiation timing in decision making (Thura and

30 Cisek, 2017; Yau et al., 2020). Well-learned action sequences may have to be initiated at precise times to

31 obtain the desired outcome, however, they may be difficult to withhold when triggering cues are present,

32 and difficult to stop once they have been initiated (Dalley and Robbins, 2017; Gillan et al., 2016a;

33 Graybiel, 2008; Knowlton and Patterson, 2016; Robbins and Costa, 2017). Moreover, in neuropsychiatric

34 conditions involving malfunctioning of cortico-basal ganglia circuits, like attention deficit hyperactivity

35 disorder, Tourette syndrome, obsessive compulsive disorder and drug addiction (Dalley and Robbins,

36 2017; Gillan et al., 2016a; Singer, 2016) action sequences might be started at inappropriate contexts and

37 timings. However, how the striatum contributes to action sequence initiation timing remains poorly

38 understood.

Interestingly, impulsivity has been identified as a vulnerability factor for compulsive drug seeking

40 habits (Belin and Everitt, 2008). Recent studies link impulsivity to automaticity in behavior (Ersche et al., 
41 2019; Gillan et al., 2016b; Hogarth et al., 2012) and to a preponderance of habitual over goal-directed

42 behavioral control (Everitt et al., 2008; Voon et al., 2015). An influential theory postulates a dual control

43 system for behavior, where dorsolateral striatal (DLS) circuits support habitual stimulus-response control

44 whereas dorsomedial striatal (DMS) circuits mediate cognitive-based deliberative control (Balleine and

45 Dickinson, 1998; Daw et al., 2005; Graybiel, 2008; Yin and Knowlton, 2006). During action sequence

46 learning, neuronal activity in the DLS rapidly evolves to mark the initiation and termination of the

47 acquired sequence (Jin and Costa, 2010; Jog, 1999), possibly contributing to its release as a behavioral

48 unit or chunk (Graybiel, 2008). By contrast, the DMS encodes reward expectancy, reward prediction

49 errors and trial outcomes even after extensive training (Kim et al., 2009; Kubota et al., 2009; Rueda-

50 Orozco and Robbe, 2015; Samejima et al., 2005; Thorn et al., 2010; Vandaele et al., 2019), likely

51 contributing to the regulation of explore-exploit (Barnes et al., 2005), cost-benefit (Floresco et al., 2008;

52 Schultz, 2015) and speed-accuracy (Thura and Cisek, 2017) tradeoffs during decision making. A bias

53 towards exploration and risk taking (Addicott et al., 2017), low tolerance to delayed rewards (Dalley and

54 Robbins, 2017; Monterosso and Ainslie, 1999; Wittmann and Paulus, 2008) and elevated internal urgency

55 states (Carland et al., 2019), may all contribute to impulsivity traits. However, how neuronal activity in the

56 dorsal striatum encodes action sequence initiation timing, and whether this encoding informs about

57 enhanced premature responding in conditions of high impulsivity, remains to be comprehended.

Here we studied striatal activity during a self-paced task where rats have to withhold a rewarded

59 action sequence until a waiting interval has elapsed (Zold and Hussain Shuler, 2015). Prematurely initiated

60 sequences were penalized by re-initiating the waiting interval; however, the animals showed premature

61 responding even after extensive training and often failed to interrupt sequence execution despite sensory

62 evidence of its untimely initiation. Thus, the task allowed comparing striatal activity during behaviorally

63 indistinguishable prematurely and timely executed learned action sequences. We found that a peak of

64 striatal activity preceding trial initiation was modulated by time waited before responding. Moreover, this 
65 modulation grew at a faster rate in adolescent rats, likely reflecting a steepest reward expectancy increase

66 during waiting that paralleled their more impulsive behavior compared to adult rats.

\section{Results}

Rats learn to make timely action sequences to obtain a water reward

Water-deprived rats were trained to obtain water from a lick tube located within a nose-poke by

70 emitting a sequence of eight licks following a visual cue (Figure 1a and b; task modified from Zold and

71 Hussain Shuler,2015). Trials were self-initiated by the animal by entering into the nose-poke. In those

72 trials initiated $2.5 \mathrm{~s}$ after the end of the previous trial (timely trials), a $100 \mathrm{~ms}$ duration visual cue (two

73 symmetrical green LEDs located at the nose-poke sides) reported that there was a 0.5 probability of

74 receiving a water reward. Prematurely initiated trials were penalized by re-initiating the waiting interval.

75 Spike discharges and local field potentials (LFP) were recorded from the dorsal striatum using custom

76 made tetrodes (Vandecasteele et al., 2012) (representative localization Figure 1c, for detailed localization

77 see Supplementary Figure 10).

Adult rats learned to make timely nose-poke entries followed by an 8-lick sequence (Figure 1d), as

79 evidenced by a twofold higher reward rate late in training (after three consecutive sessions with $>70 \%$

80 correct trials) than early in training (Figure 1e, $\mathrm{p}<0.0001$, Wilcoxon matched pairs test). Performance

81 became faster with training (Figure 1f-h): trial duration $(p=0.0005)$, latency to the first lick $(p=0.0032)$ and

82 time to complete the 8-lick sequence $(p=0.0008)$, diminished with training for both rewarded and

83 unrewarded timely trials (significant effect of learning stage, non-significant interaction, two-way RM-

84 ANOVAs).

Premature nose-poke entries delayed the opportunity to get the reward as evidenced by a negative

86 correlation between the relative number of premature trials and reward rate across sessions (Figure 2a). 
87 Premature trials diminished with training from $>30 \%$ of all trials at the beginning of training to $~ 15 \%$ at

88 the end of training. However, premature trials followed by an 8-lick sequence rose from $40 \%$ to $70 \%$ of all

89 premature trials with training, paralleling the relative increase of 8-lick sequences observed in timely trials

90 (Figure 2b, $\mathrm{p}<0.0001$ for learning stage and trial timing, no interaction, two-way RM-ANOVA). These

91 data suggested that behavior during premature trials was modified by learning. Further supporting this

92 presumption, time to complete the 8-lick sequence $(\mathrm{p}=0.0014)$, latency to the first lick of the sequence

$93(p=0.002)$ and variation coefficient of the inter-lick intervals (an index of regularity of such intervals;

$94 \mathrm{p}=0.021$ ), diminished with training both for timely and premature trials (Figure 2c-e; significant effect of

95 learning stage, no effect of trial type, no interaction, two-way RM-ANOVA). Remarkably, even though

96 premature trials had a negative effect on reward rate (Figure 2a), there was a significant positive

97 correlation between the percentage of premature trials followed by an 8-lick sequence and reward rate

98 (Figure 2f).

To characterize the timing of trial initiations in adult rats, plots showing the frequency distribution 100 of all trial initiation times were built (Figure 2g-h). The probability of a trial including an 8-lick sequence 101 sharply increased at the end of the $2.5 \mathrm{~s}$ waiting interval, peaked immediately after its finalization, and 102 then diminished gradually. Similar results were observed in a separate group of rats trained with a longer 103 waiting interval (Supplementary Figure 1a-h). Finally, rats trained with the long waiting interval (5 s) 104 quickly learned to adjust trial initiations to a shorter waiting interval (2.5 s) (Figure 2i), suggesting that 105 premature trials with 8-lick sequences served to adapt behavior to changes in the waiting time 106 requirements of the task that otherwise would have passed unnoticed to the rats.

An additional group of adult rats was trained with a modified version of the task that required 108 initiating trials not before $2.5 \mathrm{~s}$ and no later than $5 \mathrm{~s}$ after exiting the port in the previous trial. These 109 animals showed similar behavior with a more marked decrease of trial initiations after the peak rate 110 observed at $2.5 \mathrm{~s}$ was passed (see below; Supplementary Figure 2). 
111 Altogether, the data show that adult rats optimized reward rate by waiting the least possible time

112 between trials. Noteworthy, premature execution of the learned behavioral response was (relatively) more

113 common late in training than early in training, suggesting that with training behavior became less sensitive

114 to the absence of the reward predictive visual cue.

115 Task-sensitive striatal activity concentrates at the boundaries of the learned behavioral response

116 To determine if striatal activity marks the boundaries of the learned action sequence in our task (Jin

117 and Costa, 2010; Jog, 1999), DMS activity recorded from adult rats was analyzed by aligning the activity

118 to port entry and port exit (Figure 3a-c). Visual inspection of neuronal raster plots and peri-event time

119 histograms (PETH) showed strong modulations of DMS activity preceding port entry and/or at the time of

120 port exit, during timely trials (Figure 3a-b). Overall, 50\% of the recorded units (n=867) showed higher

121 activity (>1 SD over baseline) preceding port entry (“anticipatory activity”; 19\%, Figure 3d), at the time

122 of port exit (18\%, Figure 3e) or both before port entry and at port exit (12\%, Figure 3f). On average, these

123 neurons showed lower than baseline firing rates during the execution of the learned action sequence

124 (Figure 3d-f). There were also neurons ( $\mathrm{n}=105,12 \%$ of all recorded neurons) showing higher activity

125 when the animal was inside the port than during the waiting period or at the initiation and finalization of

126 the action sequence (Figure 3g). Finally, 82 neurons classified as non-task responsive were tonically active

127 during the waiting period regardless of the waited time (Supplementary Figure 3a). All main types of task-

128 related activity emerged early during training (Supplementary Figure 3b).

Thus, although DMS activity was continuously modulated during the present task, modulations at

130 the boundaries of the behavioral response accounted for about 50\% of all task related activity and more

131 than $30 \%$ of the recorded neurons showed a peak of activity anticipating trial initiation. 


\section{2}

133

134

135 additional factors, like reward anticipation and the vigor and value of the upcoming action (Lauwereyns et

136 al., 2002; Samejima et al., 2005; Wang et al., 2013). Moreover, it has been proposed that changes in

137 striatal activity preceding the initiation of a prepotent action may predict premature responding (Buckholtz

138 et al., 2010; Donnelly et al., 2014; Wu et al., 2018). Because in the present task an automatized action

139 sequence is often prematurely released, we asked if the observed anticipatory activity specifically predicts

140 the release of the learned action sequence, and if, additionally or alternatively, it encodes its timing. When

141 all port entry responsive neurons were considered (i.e., port entry only plus port entry/port exit neurons),

142 the average firing rate modulation anticipating trial initiation was higher for timely than for premature

143 trials irrespective of the upcoming action including the 8-lick sequence or not (Figure 4a-b; $<<0.0001$,

144 significant main effect of trial initiation timing, no effect of action sequence structure, no interaction, two

145 way RM-ANOVA). On average, this activity began $1 \mathrm{~s}$ before and peaked $0.5 \mathrm{~s}$ before the animal crossed

146 the infrared beam located at port entry, both in premature and timely trials (Figure 4a). Further data

147 analysis showed that this modulation of striatal activity by trial initiation timing was independent of

148 electrode location within the DMS and involved both port entry only and port entry/port exit neurons

149 (Supplementary Figure 4a-b). Since this anticipatory activity closely preceded approaching movements

150 towards the nose-poke, we analyzed accelerometer recordings of head movements performed during the

151 task. The accelerometer recordings did not differ between premature and timely trials (Figure 4c).

152 Furthermore, the 8-lick sequences emitted during premature and timely trials lasted the same and had the

153 same latency and inter-lick interval regularity (Figure 2c-e), suggesting similar action vigor during

154 premature and timely 8-lick trials. Thus, in this task, the firing rate modulation preceding trial initiation

155 discriminates between premature and timely trials and does not predict the speed, regularity, structure, 
156 value or vigor of the subsequently released action sequence.

To further investigate this anticipatory activity, we plotted its amplitude at increasing waiting times

158 observing that it increased with a steep slope as time waited surpassed the learned waiting interval and

159 then plateaued (Figure 4d-e). Similar results were obtained in rats trained with a longer waiting interval

160 (Supplementary Figure 5). Contrastingly, no modulation of this same neuronal activity by time waited in

161 the following trial (Figure 4f), or of port exit related activity by the preceding waiting time (Figure 4g),

162 was observed. The steep slope of the curve at the criterion waiting interval suggested that the neuronal

163 activity does not linearly report elapsed time but rather changes in reward anticipation as waiting

164 progressed. To explore this possibility further, we analyzed striatal activity of rats trained with a modified

165 version of the task requiring initiating trials not before $2.5 \mathrm{~s}$ and no later than $5 \mathrm{~s}$ after exiting the port in

166 the previous trial. Waiting less than $2.5 \mathrm{~s}$ (premature trials) or more than $5 \mathrm{~s}$ (late trials) was penalized by

167 resetting the waiting interval (Figure 4h). We reasoned that if this activity provides a wait time-based

168 reward anticipation signal for the upcoming action, it should decrease (instead of plateauing) after $5 \mathrm{~s}$ of

169 waiting in the modified version of the task. As in the standard version of the task, the animals learned to

170 wait the less possible time between trials, and also noticed the effect of the cutoff time on reward

171 probability, as evidenced by a reduced number of late trials with training (Figure 4i, $\mathrm{p}<0.0001$ versus

172 timely trials, Tukey post hoc test after significant one-way RM-ANOVA; Supplementary Figure 2). The

173 firing rate modulation preceding trial initiation increased with a steep slope at $2.5 \mathrm{~s}$, confirming results

174 obtained with the basic version of the task, but instead of plateauing, it decreased after surpassing the $5 \mathrm{~s}$

175 cutoff time, yielding a significant interaction in a two way ANOVA comparing the effects of waiting time

176 on anticipatory activity (Figure $4 \mathrm{j}$ and $4 \mathrm{k}$, significant effect of training, $\mathrm{p}=0.033$, trials, $\mathrm{p}<0.0001$, and

177 interaction, two-way RM-ANOVA).

In summary, the marked modulation of striatal activity preceding trial initiation probably reflects

179 subjective changes in reward anticipation as waiting progressed. 
Trial initiation timing modulates striatal activity at predicted outcome time

181

Striatal activity can be modulated by reward-predictive sensory cues (Schultz, 2015). In the present

182 task, a small population of neurons whose activity was modulated at the time of the visual cue $(n=27,3 \%$

183 of all recorded neurons) showed lower activity during premature trials, when the visual cue was not

184 presented ( $\mathrm{p}<0.05$ versus timely trials, Tukey post hoc test after significant one-way RM ANOVA)

185 (Supplementary Figure 6). This modulation by trial initiation timing was similar to that observed in port

186 entry neurons and may represent the same kind of wait time-based reward anticipation activity that

187 extends until when sensory feedback discloses if reward could be obtained or not. In contrast, neurons

188 showing increased activity during licking did not show any modulation by trial initiation timing (Figure 5a

189 and 5b). Detection of activations occurring during narrow time windows centered over individual licks

190 yielded largely overlapping populations of positively modulated neurons with broad activity peaks

191 encompassing many inter-lick intervals (Figure 5b). This modulation lasted longer during rewarded trials

192 (where licking also persisted for longer) than during timely unrewarded and premature trials, but was of

193 similar amplitude at the time when reward was expected irrespective of reward delivery or omission

194 (Figure 5b, activity centered at the eight lick), suggesting that these neurons were modulated by licking.

195 Striatal activity is also modulated by trial outcome and distinguishes between rewarded and non-

196 rewarded trials in probabilistic tasks (Atallah et al., 2014; Histed et al., 2009; Nonomura et al., 2018; Shin

197 et al., 2018; Yamada et al., 2011). In the present task, premature 8-lick trials seem to be necessary to

198 maintain an internal representation of the criterion waiting interval. To determine if the negative outcome

199 associated to premature 8-lick trials is reported by striatal neurons, we looked for outcome-related activity

200 modulations at the time of the eight lick during timely rewarded, timely unrewarded and premature trials

201 with complete sequences (Figure 5c-g). Overall, 15\% of the neurons recorded in adult rats (125 out of

202 867) showed responses at the time of the 8-lick that differed between these trial types. Five percent of all 
203 recorded neurons $(n=45)$ showed a higher activity modulation when reward was obtained than in the no-

204 reward conditions (Figure 5c, f top - reward responsive neurons, $\mathrm{p}<0.0001$, Tukey test after significant 205 one-way RM ANOVA), before lick rates began to diverge by trial type (Figure 5h). Moreover, 5\% of the 206 striatal neurons showed activations at the time of the no-reward outcome in timely unrewarded trials and 207 were unresponsive to reward delivery (Figure 5d). Interestingly, these neurons also showed a marked 208 activation at the time of the 8-lick during premature 8-lick trials (Figure 5d, f middle - no-reward 209 responsive neurons, $\mathrm{p}<0.0001$ versus rewarded trials, Tukey test after significant one-way RM ANOVA).

210 Strikingly, there were 33 neurons (4\% of all recorded neurons) that showed a two-fold higher activation at 211 the time of the eight lick in premature trials than in timely unrewarded trials (Figure 5e, f bottom; $212 \mathrm{p}<0.0001$, Tukey test after significant one-way RM ANOVA). This difference cannot be explained by 213 differences in lick rates because licking decreased at similar rates after the 8-lick in both types of non214 rewarded trials (Figure 5h). Further data analysis showed that, indeed, the three populations of outcome 215 responsive neurons discriminated timely unrewarded from premature 8-lick trials; however, those showing 216 a higher activation during premature trials discriminated better between these two no-reward conditions 217 (Figure 5g). Overall, the data supports that activity at the time of expected trial outcome reflects 218 differences in reward prediction between premature and timely trials.

\section{Activity at port exit reports whether the animal has performed the learned action sequence or not}

Although striatal activity predominated at the boundaries of the learned behavioral response, the

221 activity preceding response initiation was not invariably connected to the execution of the learned action 222 sequence. However, when all port exit neurons were considered (i.e., port exit only plus port entry/port 223 exit neurons), a higher activity was observed for 8-lick trials than for trials with an incomplete licking 224 sequence, independently of whether the trials were timely initiated or not (Figure 6a-b; $\mathrm{p}<0.001$ for lick 225 sequence structure, no effect of trial timing, no interaction, two way RM ANOVA). Discrimination of 
226 incomplete lick sequence trials was more marked in the port exit only neurons and was independent of

227 striatal recording site (Supplementary Figure 4c-d). Moreover, there was no between trials difference in

228 the accelerometer data at the time animals exited the port (Figure 6c). Thus, port exit related activity

229 seems to tell if the learned response was emitted or not regardless of its timing and outcome.

\section{Adolescent rats make more premature trials}

Rats implanted with tetrodes 30-35 days after birth learned the $2.5 \mathrm{~s}$ waiting interval task as shown

232 by a higher percentage or correct trials (Figure 7a), a higher reward rate (Figure 7b, *p<0.0001, Wilcoxon

233 matched pairs test), and a faster performance with training (Supplementary Figure 7). Moreover, trial

234 duration, latency to first lick and 8-lick sequence duration did not differ between adolescent and adult rats

235 (Supplementary Figure 7a-c), nor between premature 8-lick and timely 8-lick trials in adolescent rats

236 (Supplementary Figure 7e-g). Finally, the proportion of premature 8-lick sequence trials increased with

237 training in adolescent rats (Figure 7c; $\mathrm{p}<0.0001$, significant effects of learning stage and trial timing, no

238 interaction, two-way RM ANOVA), in parallel with reward rate (Figure 7d), and the frequency distribution

239 of trial initiation times showed that adolescent rats learned the waiting interval (Figure 7e). In summary,

240 adolescent rats also released the learned action sequence prematurely despite extensive training, and

241 indeed, they did it more frequently than adult rats (Figure $7 f$ and $7 \mathrm{~g}$ ). The number of premature trials per

242 reward obtained was twofold higher in adolescent than adult rats even after training (Figure 7f; $p=0.004$,

243 significant effects of age group, training, and interaction, two-way RM ANOVA), and this was due to a

244 preferential retention of the premature 8-lick trials through training (Figure 7g; $\mathrm{p}=0.0024$, significant

245 effect of age group, no effect of training, no interaction, two-way repeated measures ANOVA). Finally, a

246 comparison of the cumulative frequency distributions of 8-lick trial initiation times at late stages of

247 training showed that the relative excess of premature 8-lick trials observed in adolescent rats parallels an

248 excess of late (yet rewarded at $\mathrm{p}=0.5$ ) trials in adult rats (Figure $7 \mathrm{~h}$ ), suggesting that adult rats are more 
249 tolerant to reward delay. Altogether, adolescent rats achieved the same reward rate as adult rats but at the 250 expense of a higher cost as evidenced by the excess of premature responses per reward obtained.

251 Steepest reward anticipation signal preceding trial initiation in adolescent rats

252 Overall, task-related striatal activity was qualitatively similar in adolescent and adult rats 253 (Supplementary Figure 8). Of the registered units in adolescents $(n=552)$, the proportion of neurons 254 showing port entry, port exit and lick-related activity was similar to that found in adult rats (Figure 7i; $255 \mathrm{p}=0.83$, chi square test). Also like in adult rats, the activations preceding port entry were higher for timely 256 than for premature trials regardless of whether the 8-lick sequence was completed or not (Supplementary 257 Figure 9). Outcome-related activations discriminating reward delivery from reward omission, and 258 selective activations at expected reward time during premature 8-lick trials, not attributable to differences 259 in lick rate, were also present (Supplementary Figure 9).

We therefore looked for quantitative differences in task-related activities that could account for the 261 more impulsive behavior of adolescent rats. Data from three adolescent and five adult rats (133 and 103 262 DMS neurons with anticipatory activity, respectively) trained in the $2.5 \mathrm{~s}$ waiting interval task were used 263 for the following analysis. Curves displaying the amplitude modulation of activity preceding trial 264 initiations at increasing waiting times, probably reflecting reward anticipation, showed a steepest increase 265 in adolescent rats as the criterion waiting interval was surpassed (Figure 7j). When all port entry 266 modulated neurons were considered, statistical comparisons based on a general linear model showed a 267 significantly higher activity modulation by waiting time in adolescent rats (significant effect of age, $268 \mathrm{p}<0.001$, and waiting time, $<0.001$, no interaction). On the other hand, activations at time of expected 269 reward (Figure $7 \mathrm{k}, \mathrm{p}<0.0001$, effect on trial initiation timing, Restricted Maximum Likelihood test, 270 REML) and activity at port exit, probably reflecting that the correct action sequence was emitted (Figure 271 7l, $\mathrm{p}<0.0001$, significant interaction between trial initiation timing and sequence structure, three-way 
272 ANOVA), did not differ between adolescent and adult rats.

273 In sum, the data show that the waiting modulation of reward anticipation grows at a faster rate in

274 adolescent rats.

\section{Discussion}

In the present task, water-deprived rats learn to withhold a prepotent response to a water port to

277 avoid a negative contingency, the re-initiation of the waiting interval, which delays the opportunity to get

278 the next reward. The task resembles differential reinforcement of low rates of responding protocols (DRL)

279 that are used to assess response inhibition and timing factors associated with impulsivity (Monterosso and 280 Ainslie, 1999; Neill, 1976). Factors that increase premature responding in humans, such as 281 psychostimulant drugs (Evenden, 1998), psychostimulant withdrawal (Peterson et al., 2003), D2-type 282 receptor agonists (Engeln et al., 2016), maternal separation and social isolation (Lovic et al., 2011), sleep 283 restriction (Kamphuis et al., 2017) and adolescence (Andrzejewski et al., 2011) also increase premature 284 responding in rodents trained with DRL procedures. Furthermore, these procedures have been used in 285 educational and clinically meaningful contexts to reduce impulsive behavior (Bonner and Borrero, 2018;

286 Lennox et al., 1987). Unlike classical DRL procedures, the present task requires responding with an action 287 sequence that becomes highly automatized with training and could not always be stopped despite feedback 288 telling that it will not be rewarded. Interestingly, although premature responding showed an overall 289 decrease with training, the premature releases of the learned action sequence were selectively preserved, 290 suggesting that these responses are necessary to learn the duration of the waiting interval and to adapt to 291 its changes. As long as the waiting interval remains predictable, investigation of the waiting interval 292 through premature responding should be minimized to improve reward rate. Importantly, adolescent rats 293 learn the task but make more premature responses per reward obtained, which makes them more 294 impulsive than adults. 
Recent studies link premature responding in anticipation of reinforcement to a higher tendency to

296 automatize behavior and form habits (Everitt et al., 2008; Voon et al., 2015). Striatal activity marking the

297 boundaries of automatized action chains has been perceived as a signature of "packaged behavioral

298 sequences" (Graybiel, 2008) that would be difficult to stop after their release regardless of whether their

299 initiation was more or less goal-directed (Geddes et al., 2018; Robbins and Costa, 2017). While this

300 "bracketing activity" prevails in the DLS, representation of task events contributing to goal-directed

301 behavior persists in the DMS even after extensive training (Kubota et al., 2009; Thorn et al., 2010) . These

302 parallel representations may allow switching between automatic and deliberation-based task-solving

303 strategies when outcomes change (Balleine and Dickinson, 1998; Daw et al., 2005; Yin and Knowlton,

304 2006) or interventions impair behavioral control by one of the circuits involved (Gremel and Costa, 2013;

305 Smith and Graybiel, 2013; Yin et al., 2004). The remarkably similar behavior observed in 8-lick

306 prematurely released and timely unrewarded trials led us to expect that a stronger boundary activity,

307 and/or a less precise coding of task events, could explain the higher rates of premature responding

308 observed in adolescent rats. Indeed, a recent study suggested that the stronger the "opening” activity, the

309 lower the deliberation at the turn-choice site in a T-maze task (Smith and Graybiel, 2013). Here, the

310 strength of anticipatory activity increased with the time waited before response release and was higher in

311 the more impulsive adolescent rats. However, it did not predict that the rewarded 8-lick sequence would

312 be included in the behavioral response. On the other hand, a similar closing activity followed all responses

313 containing the learned 8-lick sequence regardless of trial outcome and despite the markedly different lick

314 rates observed between rewarded and unrewarded responses after outcome disclosure. A recent study

315 showed that hierarchical control during action sequence execution may allow the selective removal of

316 intermediate sequence elements (Geddes et al., 2018), which could be a mechanism through which licks

317 could be deleted from behavioral responses preceded by similar wait time-based reward expectations in

318 the present task. Moreover, since our recordings were obtained from the DMS, we cannot rule out that a 319 different opening activity specific for the learned sequence emerges in the DLS in our task as previously 
320 reported for other tasks (Jin and Costa, 2010; Jog, 1999; Martiros et al., 2018; Thorn et al., 2010).

321 Importantly, while striatal neurons with opening and/or closing activity were poorly modulated during 322 sequence execution, as shown in other studies (Jin and Costa, 2010; Jog, 1999), the activity of many 323 striatal neurons seemed to continuously follow the expression of the lick sequence as has also been 324 reported previously by others (Jin and Costa, 2010; Jin et al., 2014; Rueda-Orozco and Robbe, 2015). 325 Overall, our data show that the DMS expresses opening/closing activity even after skilled performance is 326 reached, as reported in a recent study with a different task (Vandaele et al., 2019), and suggest that, at least 327 in the DMS, this activity is more flexible regarding the properties of the behavioral response it bounds, 328 and carries information about its pertinent timing.

We speculated that the more impulsive behavior of adolescent rats observed in the present task 330 could relate to changes in wait time-based reward anticipation signals and/or outcome evaluation signals.

331 Striatal signals at the time of expected outcome not only discriminated between rewarded and unrewarded 332 trials as observed by others (Atallah et al., 2014; Nonomura et al., 2018; Shin et al., 2018), but also 333 premature from timely responses. A specific outcome evaluation signal after premature 8-lick trials may 334 serve to minimize the exploration of the waiting interval. To compute such outcome signal, the animal has 335 to retain information regarding the time waited before responding (or regarding visual feedback on proper 336 trial initiation timing) while monitoring the execution of the lick sequence. Such kind of integration could 337 be implemented by retaining information of early task events along the sequential activation of striatal 338 ensembles (Her et al., 2016; Nonomura et al., 2018). However, although the effect of waiting time on task339 related striatal activity extended until the time of the visual cue, it was absent in ensembles with lick340 related activity. Thus, the sustained lick-related signal may serve to estimate outcome timing (Zold and 341 Hussain Shuler, 2015), but information about trial initiation timing should arrive to outcome-sensitive 342 ensembles through other mechanisms likely involving inputs from prefrontal and orbitofrontal cortex 343 areas (Asaad et al., 2017; Hamid et al., 2021; Wassum et al., 2011). Nonetheless, we found no differences 
344 in outcome evaluation signals that could explain the more impulsive behavior of adolescent rats. On the

345 other hand, although premature responding has classically been linked to poor behavioral inhibition (Bari 346 and Robbins, 2013), alternative views equates it to preferring a smaller more immediate reward over a 347 larger delayed one (Dalley and Robbins, 2017; Monterosso and Ainslie, 1999), and to deficient 348 accumulation or evaluation of evidence while waiting (Dalley and Robbins, 2017). Interestingly, 349 influential models propose that time estimates derived from accumulation of pacemaker counts can be 350 compared to a memorized time interval to decide whether a target time has been reached (Buhusi and 351 Meck, 2005; Gibbon, 1977; Namboodiri and Hussain Shuler, 2016). Previous studies showed that 352 sequentially activated striatal ensembles can provide time estimations (Bakhurin et al., 2017; Emmons et 353 al., 2017; Gouvêa et al., 2015; Matell et al., 2003; Mello et al., 2015; Zhou et al., 2020) and we also found 354 sequential and tonic ensemble activation during the waiting period (Figure 3b and Supplementary Figure 355 3a) that could serve to track time while waiting in the present task. By comparing the time accumulated 356 while waiting against the memorized waiting interval, the animal could anticipate how likely is the 357 upcoming action to be rewarded. How fast reward expectancy steps up when time waited approaches the 358 reference time interval would depend on several factors, including temporal discounting effects on reward 359 value (Monterosso and Ainslie, 1999; Namboodiri and Hussain Shuler, 2016; Wittmann and Paulus, 360 2008). In this sense, the anticipatory signal we observe could be interpreted as a reading of the temporal 361 discount function at the time chosen to release the learned action sequence. A recent theory proposes that 362 animals time their decisions by estimating if they can improve the reward rate experienced in the recent 363 past; according to it, the longer the time window over which reinforcement history is estimated, the higher 364 the tolerance to delays of future rewards (i.e., the less steep the temporal discount effect) (Namboodiri et 365 al., 2014) . Adults should be able to integrate information about past reinforcement history over longer 366 time windows that adolescents (Namboodiri et al., 2014), which is consistent with the finding that 367 temporal discount rate decreases throughout childhood and adolescence (Green et al., 1999; Scheres et al., 368 2006; Steinberg et al., 2009). Alternative theories propose that waiting impulsivity relates to perceiving 
369 durations as longer as they are, which would be associated to perceiving a higher cost of time and to 370 steeper temporal discounting (Wittmann and Paulus, 2008). Moreover, time perception depends on several 371 cognitive processes that are modified by adolescence including working memory, attention and mood 372 (Baumann and Odum, 2012; Wittmann and Paulus, 2008). It has been also been proposed that impulsivity 373 relates to changes in an internal urgency signal that influences the timing of decisions and may be primary 374 responsible for the build-up of neural activity observed in the striatum and cortex that often precedes 375 action initiation (Carland et al., 2019). Interestingly, in the present task, adolescent rats achieve a similar 376 reward rate than adult rats and indeed, they do not show the negative effect of premature trials on reward 377 rate that is observed in adults (Supplementary Figure 7). Importantly, although they achieve this reward 378 rate at the expense of a higher energy cost (more premature trials per reward obtained), because most 379 premature trials remaining after reaching skilled performance correspond to the learned action sequence, 380 adolescents would likely get more precise information regarding the duration of the waiting interval and 381 would also refresh more frequently their cognitive map of the task. This view is consistent with the idea 382 that impulsivity is advantageous when it helps to adapt to uncertain environments and that increased 383 exploratory behavior in adolescence allows to gain knowledge that would be useful to guide decisions in 384 adulthood (Addicott et al., 2017; Spear, 2000). In this context, the steepness of the wait time-based reward 385 anticipation signal could be under the influence of a gain factor that, according to reinforcement learning 386 models, reflects the degree of preference for the highest value option and regulates explore-exploit trade 387 off (Addicott et al., 2017). Further studies are needed to disclose which factors influence the wait time388 based reward anticipation signal we observe in the present task and which among them are responsible for 389 the differences observed between adult and adolescent rats. 
393 time task), the present task is learned rapidly, which makes it suitable for studying premature responding

394 during rodent adolescence. Moreover, by requiring self-paced responding with a stereotyped action

395 sequence, and making uncertain reward obtention during timely trials, the task generates conditions that 396 “clamp” behavior. This allowed identifying a modulation of anticipatory striatal activity by time waited 397 before responding, which grows with a steeper slope in adolescent rats, likely reflecting age-related 398 changes in temporal discounting, internal urgency states or explore-exploit balance. Translational studies 399 are necessary to understand if similarly designed tasks capture the relationship between impulsivity and 400 automaticity in behavior that has been related to vulnerability to drug addiction.

\section{Materials and Methods}

\section{Subjects}

Subjects were adult male Long-Evans rats from our own colony (Adolescents: 4 weeks of age at

404 the beginning of the experiments, weight 120 g; Adults: 8-12 weeks of age at the beginning of the 405 experiments, weight $\sim 390 \mathrm{~g}$ ), housed on a 12:12 light:dark cycle and experiments were performed during

406 the light phase, $21^{\circ} \mathrm{C}$ room temperature. Rats were housed in groups of 3-4 in regular cages with wood 407 shavings' bedding and, after surgery, they were singly housed in $45 \mathrm{~cm} \times 35 \mathrm{~cm} \times 22 \mathrm{~cm}$ cages with 408 moderate environmental enrichment (toys, tissue paper strips) and cardboard bedding. Two days before 409 the beginning of the behavioral training subjects were deprived of water. All throughout the training 410 schedule subjects had restricted access to water for 20 min each day and a-day rest period each week. This 411 schedule maintained animals at 90\% of their predeprivation weight, with any further weight loss being 412 counteracted by increased free water access. All procedures complied with the National Institutes of 413 Health Guide for Care and Use of Laboratory Animals (Publications No. 80-23, revised 1996) and were 414 approved by the Animal Care and Use Committee of the School of Medicine of the University of Buenos 415 Aires (CICUAL). Five adult males were part of the ITI 2.5 s experiments, three adult males were part of 
416 the ITI 5s experiments (Fig. 2i and Fig. S1) and another three adult males were included in the ITI 2.5-5s

417 experiments (Fig 4h-k, Fig. S2 -all except S2f, see below-). Three adolescent male were part of ITI 2.5 s 418 experiments (Fig. 7, Fig S7-S9). Additionally two adult subjects were included in the accelerometer 419 experiment (Fig. 4 and 6) and two others were only trained with the ITI 2.5 s protocol and did not undergo 420 surgery (Fig. S2f).

\section{Electrodes}

423 M $\Omega$ impedance) were attached to a homemade micromanipulator (Vandecasteele et al., 2012). Before 424 surgery, the tetrode array was sterilized with $3 \%$ oxygen peroxide solution (AOSept ${ }^{\circledR}$ PLUS TM). The 425 array of 8 tetrodes was gradually lowered each day before training to find neuronal activity. At the end of 426 the recording session the array was also lowered to change the recording site for the next training session 427 (approximately $80 \mu \mathrm{m}$ each day). Before being implanted, the upper part of the tetrodes was painted with a 428 DII solution (SCBT, sc-213424) to facilitate the visualization of the electrodes trace before the histological 429 analysis.

\section{Surgery}

Before surgery, subjects were treated with local anaesthetic (lidocaine) in the scalp. Under deep

432 anesthesia with isoflurane (3-4\% for induction, $1-1.5 \%$ for maintenance), rats were placed in the 433 stereotaxic frame and chronically implanted with an array of tetrodes aimed at the striatum (AP: $+0.06 \mathrm{~cm}$, 434 L: $-0.25 \mathrm{~cm}, \mathrm{DV}$ : $-0.35 \mathrm{~cm}$ ). Body temperature was maintained using a heating pad. Through a small 435 craniotomy performed in the corresponding area the tetrodes were then lowered to a depth of $3.5 \mathrm{~mm}$. The 436 micromanipulator was fixed in place with dental cement. Two stainless steel screws (0-80 X 1/8” Philips 
437 Pan head) were inserted above the cerebellum to be used as ground and reference. Three additional steel

438 screws were inserted to anchor the whole implant to the skull. The craniotomy was covered with a sterile $43950-50$ mixture of mineral oil and paraffin. Towards the end of the surgical procedure, animals were treated 440 with antibiotics (i.m., enrofloxacin $10 \mathrm{mg} / \mathrm{kg}$ ) and a veterinary ointment (antiseptic, anti-inflammatory and 441 anesthetic) was applied on the skin in contact with the external side of the implant to prevent microbial 442 infections. Subjects were kept under careful observation until awakening. The first recording session 443 followed 7 days of post-surgical recovery.

\section{Behavioral training}

Rats were placed in a behavioral operant chamber that contained a 'nose-poke' at which they could

446 seek the reward by licking through a slot onto a lick tube. Breaking an infrared beam in front of the nose-

447 poke ended the inter-trial interval (ITI) and $200 \mathrm{~ms}$ after, the nose-poke was then illuminated for $100 \mathrm{~ms}$

448 (visual cue). Following the visual cue, licks were detected by breaking a second infrared beam. Upon 449 licking the required number of times (8 licks), reward ( $20 \mu \mathrm{l}$ of water) was made available on half of the 450 trials on a pseudo-random fashion. Water reward was delivered through a tube with a solenoid attached to

451 it. The behavioral task was controlled using an Arduino Uno board. Trials ended when the animal removed 452 its head from the nose-poke. Sessions ended when the subject completed 300 trials or when it was reached 453 a maximum training time of 120 min. Timely trials are those in which the subject entered the nosepoke 454 after the required minimum waiting time. Premature trials are those in which the entrance beam was 455 interrupted before having reached the minimum waiting time. Training sessions were classified into early 456 or late as follows: having the animal reached a minimum of $80 \%$ of timely trials with the complete 8457 licks' sequence for two consecutive sessions, the following sessions were considered as "late" training 458 sessions. After evaluating the total number of late training sessions, the same number of “early” sessions 459 was selected upon the first training session, i.e: having counted 3 late sessions, the first three training 
460 sessions were considered as "early" and the rest of the sessions between early and late were classified as

461 “other”. All behavioral data is expressed as mean \pm SEM (except for reward rate figures, where the 462 median is indicated, and Waiting time curves). Each dot corresponds to the average value of a session 463 from a single animal.

\section{Accelerometer experiment}

465 Two rats were trained daily in the nose-poke chamber until they reached a stable good performance

466 (equivalent to "late" training sessions). Afterwards, a MPU-6050 accelerometer was chronically attached 467 to their heads following a procedure similar to the electrodes’ implantation. Briefly, four stainless steel 468 screws were inserted in the skull and the accelerometer was glued inside a cap made of a metallic mesh 469 and dental acrylic that was anchored to the skull with dental cement. Subjects were left for one week of 470 recovery before starting again with the behavioral training. Accelerometer data (sampling rate $25 \mathrm{~Hz}$ ) was 471 collected during each training session (9 sessions in total), using an Arduino UNO board connected to a 472 PC via PLX-DAQ software (Parallax Inc.).

\section{Histology}

Animals were given a lethal dose of ketamine xilazine and transcardially perfused with cold PBS 475 containing heparin (500 U/L) followed by 4\% PFA in PBS. The brain was quickly removed, postfixed in 476 PFA at $4^{\circ} \mathrm{C}$ for $2-12 \mathrm{~h}$, and placed in a $30 \%$ sucrose solution in PBS. Frozen coronal sections (50 $\left.\mu \mathrm{m}\right)$ 477 were collected with a sliding microtome, and histological verification of the electrode endpoints and 478 recording tracks was done in microscopy fluorescent pictures and later, sections were safranin-stained to 479 confirm the visualization of the tracks.

480 Electrode positioning was considered "medial” when the tracks were found between 0 and $0.28 \mathrm{~cm}$ 
481 relative to bregma and "lateral” from $0.28 \mathrm{~cm}$ onwards, considering bregma as starting point from center 482 to right (Fig. S10).

\section{QUANTIFICATION AND STATISTICAL ANALYSIS}

\section{Neural recording and data analysis}

486 available hardware and software (sampling rate $32.5 \mathrm{kHz}$, Cheetah, Neuralynx). Neurophysiological and

487 behavioral data were explored using NeuroScope (http://neuroscope.sourceforge.net; Hazan et al., 2006).

488 Spike sorting was performed automatically, using KlustaKwik http://klustawik.sourceforge.net, followed 489 by a manual adjustment of the clusters (using "Klusters" software package; http://klusters.sourceforge.net, 490 Hazan et al., 2006). After the spike sorting procedure, all data was analyzed with MATLAB software 491 using custom-built scripts. We registered a total of 867 units in adult rats: from the $2.5 \mathrm{~s}$ WT experiments 492 there were 158 with no response and 244 were task responsive, from the $5 \mathrm{~s}$ WT experiments there were 49345 with no response and 47 were task responsive and from the 2.5-5 s WT experiments there were 79 with 494 no response and 294 were task responsive. In adolescents we registered a total of 552 units, 233 with no 495 response and 319 task responsive (2.5 s WT).

Peri-event time histograms (PETH) were created using an 80 ms bin. In order to compare between

497 animals and cells each PETH was normalized using the mean and standard deviation ( (Xi - Xmean) / 498 Xsd) calculated from a PETH of each cell centered on port entry +/- 20 s. All data is presented as 499 normalized firing rate except for the peri-event examples. Only for display purposes, PETH were 500 smoothed using a 5 points moving window.

501 In order to analyze the activity of each neuron for the different waiting times (WT), PETH were 
502 constructed using trials with similar WT. The WT intervals limits were determined to include a similar 503 number of port entries in each segment. Only $2.5 \mathrm{~s}$ and $5 \mathrm{~s}$ were fixed. The intervals used were (beginning, 504 end; in ms): [451,1915; 1916,2499; 2500,3131; 3132,3803; 3804,4999; 5000,6084; 6085,8421; 505 8422,12621; 12622,22013; 22014,1710755]. Trials with WT shorter than 450 ms were not included in the 506 analysis.

507 A neuron was considered to be responsive to the port entry if the mean of the normalized firing 508 rate $(\mathrm{mnFR})$ was greater by $1 \mathrm{SD}$ to the mean. The mnFR was computed between 640 and 80 ms prior to 509 the port entry. Similarly, a cell was considered to be responsive to the port exit if its mnFR between 240 510 ms prior and $320 \mathrm{~ms}$ after port exit was greater than 1SD. A neuron was considered to be active during 511 sequence execution (inside port cells) if its mnFR was > 1SD between 400 and 1520 ms after port entry. 512 Neurons with an increase above 1 SD from port exit to $1 \mathrm{~s}$ before port entry, calculated for every waiting 513 time interval, were labelled as tonically active. Visual cue responding cells were those whose mnFR was > 514 2SD between 160-400ms post port entry in those trials that had the visual cue. A neuron was included as 515 active around lick number 3 or 8 if their mnFR was $>1$ SD around the event (+/- 250ms). Neurons with 516 activity related to the reward were selected as follow: 1) their mnFR between 160-560 ms after the 8th lick 517 should be greater to the activity during licking (-640 to -160 ms prior to 8th lick) by 0.5 SD and 2) their 518 mnFR during 160-560 ms after the 8th lick was above 2 SD or rewarded timely trials or during 519 unrewarded timely trials. We divided these neurons in three groups: a) with response in trials with reward 520 (Fig. 5c), b) with response in timely trials with no reward, and activity higher in those trials than in timely 521 rewarded trials by $1.5 \mathrm{SD}$ (Fig. 5d), c) with response in premature trials not included in b (Fig. 5e). 522 Relative mean normalized FR was calculated for each neuron considering average FR equal to 1 for the 523 timely trials between 2,5 and $5 \mathrm{~s}$ and timely trials for the modified version of the task (Fig. 4k). 524 Discrimination index for each selection of neurons was calculated as: absolute value of (mnFR in 525 Unrewarded trials - mnFR in Premature trials, Figure 6g). 


\section{Behavioral analysis}

527 All data was analyzed with MATLAB software using custom-built scripts. Waiting time 528 histograms were calculated from the total number of entries (either early or late in training, with or 529 without the 8-licks sequence) using 100 ms bins, normalized by its maximum so that the peak of the curve 530 corresponded to a value of 1 . Curves were smoothed with a 4 bin span. For the accelerometer recordings

531 PETH were created using a $40 \mathrm{~ms}$ bin. Data collected from both animals was pooled and the normalized 532 mean and 95 CI were calculated around the entry or exit port event +/- 2.5 s. Licks PETHs were 533 constructed centered on port entry using a $10 \mathrm{~ms}$ bin and normalized to the mean and standard deviation of 534 each session. All data is presented as normalized number of licks +/- SEM.

\section{Statistical analysis}

536 Statistical analysis of behavioral data was performed with Prism 8, Graphpad Software. All figures 537 were made colorblind safe using palettes from ColorBrewer 2.0 (http://colorbrewer2.org). All early vs. late 538 comparisons were done with repeated measures two-way ANOVA, except for reward rate in which 539 Wilcoxon matched pairs test was used. To compare neuronal activity between Rewarded, Unrewarded and 540 Premature groups, repeated measures one-way ANOVA was used. To compare licking activity between 541 Rewarded, Unrewarded and Premature trials Mixed-effects model (Restricted Maximum Likelihood test, 542 REML) was used. The performance of adults trained with a $5 \mathrm{~s}$ criterion time was analyzed at the late 543 stage of training using Mann Whitney Test. Localization of the neurons and their activity at the entry or 544 exit was analyzed using Three-way ANOVA. Linear regression was used to analyze premature trials and 545 the reward rate. A general linear model was used to compare neuronal activity from adult and adolescent 546 rats. Model: Neuronal response intercept + waiting time x age (as factor), using a Gaussian distribution. 


\section{Acknowledgments}

548 We thank Dr. Juan Belforte for helpful discussions and also Yamila Páez for her technical 549 assistance with animal welfare. This project was funded with research grants from FONCYT (PICT 2016550 0396; PICT 2017-0520; PICT 2017-2465) and UBACYT (2018-2020 305BA).

\section{Author contributions}

552 Conceptualization G.M., M.B./ Methodology M.B., C.M., C.Z./ Software M.B., C.M./ Formal 553 Analysis M.B., C.M., G.M./ Investigation C.M., M.B./ Resources G.M.,M.B./ Data Curation M.B./ 554 Visualization C.M., M.B. / Writing -Original Draft G.M., C.Z./ Writing -Review \& Editing C.Z., M.B., 555 C.M./ Project Administration G.M., M.B./ Funding Acquisition G.M., M.B., C.M.

\section{Declaration of interests}

557 The authors declare no competing interests.

\section{Lead Contact}

559 Further information and requests for resources and reagents should be directed to and will be 560 fulfilled by the Lead Contact, Mariano Belluscio. All the code used for data analysis in this paper will be 561 shared by the lead contact upon written request. Data is reported in

\section{Figure Legends}


563 Figure 1. Rats become skilled in the task. (a) Training cage with the nose-poke. Animals' entries and

564 exits from the nose-poke are detected with two infra-red beams. A visual cue of 100 ms is given with a

565 pair of green LEDs placed inside the nose-poke to indicate a timely entry. (b) Schematic representation of

566 the different types of trials. Timely trials require a minimum waiting time of $2.5 \mathrm{~s}$ and Premature trials are

567 those in which the minimum waiting time is not met. After that, trials are classified by whether animals

568 performed an 8-lick sequence or not. (c) Top: Representative diagram of the electrodes’ positioning, aimed

569 at the dorsal striatum. Bottom: Histological section ( $\mathrm{AP}=0.24 \mathrm{~cm}$ from bregma) with the traces of the

570 electrodes painted with DII. (d) Percentage of the different trial types per session,timely trials with an 8-

571 lick sequence (Tx8L) or not $(\mathrm{T}<8 \mathrm{~L})$ and premature trials with an 8-lick sequence (Px8L) or not $(\mathrm{P}<8 \mathrm{~L})$.

572 (e) Reward rates for early and late training stages. Trial duration (f), latency to the first lick during correct

573 trials (g) and time to complete the 8-lick sequence (h) for the two types of correct trials (rewarded and

574 unrewarded), at each training stage.

575 Figure 2. Training does not suppress premature initiations of the learned behavioral response. a)

576 Correlation between prematurely initiated trials and reward rate $(\mathrm{Y}=-0.023 * \mathrm{X}+1.921$, slope

577 significantly different from zero $\mathrm{p}=0.0176$, $\mathrm{R}$ square 0.09031 .). Proportion of trials (b), time to complete

578 the 8-lick sequence (c), latency to first lick (d) variation coefficient (e) of the 8-lick sequence inter-lick

579 intervals for prematurely and timely initiated trials, at early and late learning stages. f) Correlation

580 between percentage of prematurely initiated trials followed by an 8-lick sequence and reward rate ( $\mathrm{Y}=$

$5810.024 * X-0.0408$, slope significantly different from zero, $\mathrm{p}<0.0001$, R square 0.5016.). g-i) Normalized

582 frequency distributions of the trial initiation times (waiting time), separated for trials with (gray) and

583 without (dotted line) 8-lick sequences, and for early (g) and late (h) training stages. Insets: percentage of

584 the trials with (gray) and without (dotted line) 8-lick sequences for each bin, zoomed around the criterion

585 time. i) Rats were trained with a $5 \mathrm{~s}$ criterion time period (blue) and afterwards were switched to a $2.5 \mathrm{~s}$ 
586 criterion time for the following two sessions (48 h after the last 5s-WT session, light blue). Dotted lines:

587 criterion time; bin size: $100 \mathrm{~ms}$; reference for normalization: bin with highest value $=1$ ).

\section{Figure 3. Striatal activity marks transitions between behavioral states of the task. a) Representative}

589 raster plots and PETH of striatal units showing firing rate modulations related to port entry and/or port 590 exit. b) Individual PETHs of all the recorded striatal neurons during correct trials, aligned to port entry 591 (left) or port exit (right), with corresponding average PETH (below). Color code for the normalized 592 activity is shown on the right. c) Proportion of neurons showing task-related firing rate modulations. d-g) 593 Individual and average PETH and average firing rates aligned to port entry (left panels) and to port exit 594 (right panels) for d) striatal neurons showing only port entry related activity, e) striatal neurons showing 595 only port exit related activity, f) striatal neurons showing activity modulations at both port entry and port 596 exit and g) striatal neurons showing higher activity while animals are inside the port. In d to g, it is 597 represented the mean (solid lines) and SE (shaded area). Colored bars over the x axis show the interval 598 used to detect firing rate modulations (red: entry, orange: exit, purple: active during the task).

599 Figure 4. Prematurely initiated trials are preceded by low anticipatory activity. (a) Average PETH of 600 entry-related neurons, during premature and timely trials, for trials with or without an 8-lick sequence 601 (diagram on top shows the types of trials analyzed). (b) Average striatal activity corresponding to the 602 PETH shown in a. (c) Accelerometer recordings of head movements around port entry for premature and 603 timely trials. It is represented the mean (solid lines) and 95 CI (shaded area). Data was obtained from a 604 total of 9 training sessions of two animals. (d) Average PETH of striatal neurons showing entry-related 605 activity around port entry sorted by waiting time duration. Color code for the intervals shown on the right. 606 (e) Mean normalized firing rate for each of the waiting time segments, 2.5 s criterion time is shown with a 607 blue hatched-line. (f) Average PETH of the same striatal neurons segmented according to next trial waiting 
608 time. (g) Average PETH of striatal neurons showing activation at port exit segmented as in (d). (h)

609 Schematic representation of the different types of trials in a modified version of the task. Timely trials:

610 waiting time $2.5-5$ s, Premature: waiting time $<2.5$ s, Late: waiting time $>5$ s. (i) Proportion of trials

611 followed by 8-lick sequences for each type of trial. (j) Average PETH of striatal neurons showing activity 612 preceding port entry according to the waiting time duration. Color code for the intervals shown on the 613 right. (k) Relative mean normalized firing rate for each of the waiting time groups for both variants of the 614 task.

615 Figure 5. Reward responsive neurons discriminate prematurely from timely initiated trials. (a) 616 Schematic representation of the different types of trials analyzed. (b) Average PETH of striatal units 617 showing firing rate modulations related to the licking activity (1st, 3rd and 8th lick). It is represented the 618 mean (solid lines) and SE. (c-e) individual and average PETHs of striatal units, aligned to 8th lick (time 0 619 s), showing positive firing rate modulations during reward delivery (c), reward omission (d) and 620 premature trials (e). (f) Mean normalized firing rate for the different trial conditions, from top to bottom: 621 reward delivery, reward omission and premature trials. (g) Discrimination index for each of the groups of 622 neurons shown in (c-e). (h) PETH showing lick rates during the three trial conditions, centered at the 8th 623 lick, with its corresponding average at the bottom. Color code for the normalized activity is shown on the 624 right.

625 Figure 6. Striatal activity at the exit reports the performance of the action sequence. (a) Average 626 PETH of neurons responding to port exit, during premature and timely trials, for trials with an 8-lick 627 sequence and trials where less than 8 licks were emitted (diagram on top shows the types of trials 628 analyzed). (b) Average striatal activity corresponding to the PETH shown in a. (c) PETH of head 629 acceleration around port exit in premature and timely trials. It is represented the mean (solid lines) and 95 
630 CI (shaded area).

631 Figure 7. Adolescent rats become skilled in the task and show a higher modulatory response. (a)

632 Percentage of the different trial types per session. (b) Reward rates for each training stage. (c) Proportion 633 of trials followed by 8-lick sequences at early and late learning stages. (d) Correlation between percentage 634 of prematurely initiated trials followed by an 8-lick sequence and reward rate $(Y=0.026 * X+0.112$, Slope 635 significantly different from zero, $\mathrm{p}<0.0001$, R square 0.7133 .). (e) Normalized frequency distributions of 636 the trial initiation times (waiting time), separated for trials with (dark gray) and without (light gray dotted 637 line) 8-lick sequences, in the late of training. Inset: percentage of the trials with and without 8-lick 638 sequences for each bin, zoomed around the criterion time. (f) Ratio between premature trials made and 639 obtained rewards for adolescents and adults. (g) Premature trials with 8-lick sequence made per obtained 640 rewards for adolescents and adults. (h) Accumulated normalized trials vs. Waiting time for adolescents 641 (mean: red, individual session: light red) and adults (mean: black, individual session: gray). (i) Percentage 642 of neurons showing task-related firing rate modulations. (j) Mean normalized FR vs. waiting time for both 643 ages. $2.5 \mathrm{~s}$ criterion time is shown with a blue hatched-line. (k) Discrimination index calculated at the time 644 of reward delivery. (I) Mean normalized FR at the time of exit for trials with or without 8-lick sequence 645 for both ages. j-l: hatched lines correspond to adolescents and full lines to adults.

\section{Supplementary Figure 1. Rats trained with a longer ITI also emit premature learned responses. (a)}

647 Reward rates for each training stage of rats trained with a 5s ITI requirement Wilcoxon matched-pairs 648 signed rank test $* * \mathrm{p}=0.0078$. (b) Proportion of trials followed by 8-lick sequences at early and late 649 learning stages, Two-way RM ANOVA, Type of trial *** $\mathrm{p}<0.0001$, Interaction Training stage $\mathrm{x}$ Type of 650 trial * $\mathrm{p}=0.0535$. (c) Time to complete the 8-lick sequence, Mann Whitney Test $\mathrm{p}=0.4894$. (d) Latency to 651 first lick for prematurely and timely initiated trials, at early and late learning stages, Mann Whitney Test 
$652 p=0.8633$. (e) Variation coefficient of the 8-lick sequence inter-lick intervals for prematurely and timely

653 initiated trials, at early and late learning stages, Mann Whitney Test NS p=0.8633. (f) Correlation between 654 prematurely initiated trials and reward rate, Linear regression $Y=-0.02755 * X+2.776$, Slope significantly

655 different from zero: $\mathrm{p}=0.0020$, R-square=0.3343. (g) Correlation between percentage of prematurely 656 initiated trials followed by an 8-lick sequence and reward rate, Linear regression $\mathrm{Y}=0.01321 * \mathrm{X}+0.5469$, 657 Slope significantly different from zero: $p=0.0192$, R-square=0.2080. (h) Normalized frequency 658 distributions of the trial initiation times (waiting time), separated for trials with (gray) and without (dotted 659 line) 8-lick sequences, in the late of training. Inset: percentage of the trials with (gray) and without (dotted 660 line) 8-lick sequences for each bin, zoomed around the criterion time.

661 Supplementary Figure 2. Rats also learn a task with lower and upper limits in the WT. Rats were 662 trained to enter the nosepoke within a minimum WT of $2.5 \mathrm{~s}$ and a maximum WT of 5. (a) Reward rates 663 for the 2.5-5 s WT task, *** $\mathrm{p}<0.0001$ Wilcoxon Signed rank test. (b) Trial duration for correct trials, 664 Paired t test ${ }^{* * *} \mathrm{p}<0.0001$. (c) Latency to first lick for prematurely, timely and late initiated trials, One665 way RM ANOVA NS, p=0.6053. (d) Time to complete the 8-lick sequence for the different types of trials, 666 One-way RM ANOVA NS, p=0.5593. (e) Variation coefficient of the 8-lick sequence inter-lick intervals 667 for the different types of trials, One-way RM ANOVA NS, p=0.6407. (f) Normalized frequency 668 distributions of the trial initiation times (waiting time), separated for trials with 8-lick sequences in the 669 third (dark blue) and the seventh (light blue) sessions of training with a $2.5 \mathrm{~s}$ WT. Inset: area below the 670 curve calculated between 2.5 s and 5 s for sessions 3 and 4 vs. sessions 6 and 7, Mann- Whitney test, NS, $671 \mathrm{p}=0.8857$. (g) Normalized frequency distributions of the trial initiation times (waiting time), separated for 672 trials with 8-lick sequences in the third (lilac) and the seventh (purple) sessions of training with a 2.5-5 s 673 WT. Inset: area below the curve calculated between $2.5 \mathrm{~s}$ and $5 \mathrm{~s}$ for sessions 3 and 4 vs. sessions 6 and 7 , 674 Mann- Whitney test * $\mathrm{p}=0.0238$. 


\section{Supplementary Figure 3. Characteristics of the neurons registered in the 2.5 s WT task. (a) Average}

676 firing rate of striatal neurons showing tonic activity during the waiting period, aligned to port entry or port

677 exit, for different waiting times. Color code for the normalized (in units of standard deviation) activity is

678 shown on the left. (b) Proportion of different responses in both training stages from animals trained with a 6792.5 s criterion time.

680 Supplementary Figure 4. Striatal activity at the boundaries of the trials. (a) Anticipatory activity of

681 entry responsive neurons and neuronal localization. Average striatal activity shown in the right panel.

682 Three-way ANOVA, Localization $\mathrm{p}=0.0005$, Training stage $* * * \mathrm{p}<0.0001$, Licks' sequence $\mathrm{p}=0.0851$,

683 Interaction Localization $\mathrm{x}$ Training stage $* * * \mathrm{p}<0.0001$, Interaction Localization $\mathrm{x}$ Licks’ sequence $684 \mathrm{p}=0.1999$, Interaction Training stage $\mathrm{x}$ Licks’ sequence $\mathrm{p}=0.2842$, Interaction Training stage $\mathrm{x}$ Licks’ 685 sequence $\mathrm{x}$ Localization $* * * \mathrm{p}=0.0006$. (b) Anticipatory activity of entry-exit responsive neurons and 686 neuronal localization. Average striatal activity shown in the right panel. Three-way ANOVA, Localization $687 \mathrm{p}=0.1824$, Training stage ${ }^{* * *} \mathrm{p}<0.0001$, Licks’ sequence $\mathrm{p}=0.1751$, Interaction Localization $\mathrm{x}$ Training 688 stage $* * * \mathrm{p}<0.0001$, Interaction Localization $\mathrm{x}$ Licks' sequence $\mathrm{p}=0.0501$, Interaction Training stage $\mathrm{x}$

689 Licks' sequence $\mathrm{p}=0.5239$, Interaction Training stage $\mathrm{x}$ Licks' sequence $\mathrm{x}$ Localization $\mathrm{p}=0.0178$. (c)

690 Activity at port exit of exit responsive neurons and neuronal localization. Average striatal activity shown 691 in the right panel. Three-way ANOVA, Localization $\mathrm{p}=0.9256$, Training stage *** $\mathrm{p}=0.0007$, Licks’ 692 sequence $* * * \mathrm{p}<0.0001$, Interaction Localization $\mathrm{x}$ Training stage $\mathrm{p}=0.0511$, Interaction Localization $\mathrm{x}$ 693 Licks' sequence $\mathrm{p}=0.5469$, Interaction Training stage $\mathrm{x}$ Licks’ sequence $* * * \mathrm{p}<0.0001$, Interaction 694 Training stage $\mathrm{x}$ Licks' sequence $\mathrm{x}$ Localization ** $\mathrm{p}=0.0022$. (d) Activity at port exit of entry-exit 695 responsive neurons and neuronal localization. Average striatal activity shown in the right panel. Three696 way ANOVA, Localization $\mathrm{p}=0.6139$, Training stage $\mathrm{p}=0.1512$, Licks’ sequence $* * * \mathrm{p}=0.0005$, Interaction 
697 Localization $\mathrm{x}$ Training stage $\mathrm{p}=0.9700$, Interaction Localization $\mathrm{x}$ Licks' sequence $\mathrm{p}=0.6973$, Interaction 698 Training stage $\mathrm{x}$ Licks’ sequence $\mathrm{p}=0.5116$, Interaction Training stage $\mathrm{x}$ Licks' sequence $\mathrm{x}$ Localization $699 \mathrm{p}=0.6400$.

700 Supplementary Figure 5. Anticipatory activity in the 5s WT task. (a) Average firing rates of striatal 701 neurons (of rats trained with a $5 \mathrm{~s}$ criterion time) showing activity preceding the initiation of the trials 702 according to the waiting time duration. Color code for the intervals shown on the right. (b) Mean of the 703 normalized firing rate for each of the waiting time groups, calculated between -640 and -80 ms before the 704 entrance vs. waiting time, criterion time is shown with a blue hatched-line.

705 Supplementary Figure 6. Neuronal activity at the moment of the visual cue. Population PETH and 706 Average firing rates of striatal units showing positive firing rate modulations related with the visual cue. 707 Color code for the mnFR is shown on the right. Below, bar graphs showing statistical comparisons 708 between trial conditions. One-way RM ANOVA *** $\mathrm{p}<0.0001$, Tukey’s multiple comparisons test $* * *$ $709 \mathrm{p}=0.0002$ Premature vs. the other groups.

710 Supplementary Figure 7. Adolescent rats make more premature trials. (a) Trial duration for correct 711 trials at each training stage, Two-way RM ANOVA, Training stage *** $\mathrm{p}=0.0002$, Type of trial ** $712 \mathrm{p}=0.0072$. (b) Latency to the first lick during correct trials, Two-way RM ANOVA, Training stage *** $713 \mathrm{p}=0.0007$. (c) Time to complete the 8-lick sequence, Two-way RM ANOVA, Training stage $* * * \mathrm{p}<0.0001$. 714 (d) Correlation between prematurely initiated trials and reward rate, Linear regression $\mathrm{Y}=-0.002263 * \mathrm{X}$ $715+1.504$, Slope significantly different from zero: $p=0.8492$, R-square $=0.0077$. (e) Time to complete the 8716 lick sequence, Two-way RM ANOVA, Training stage *** $\mathrm{p}=0.0005$. (f) Latency to first lick for 
717 prematurely and timely initiated trials, at early and late learning stages, Two-way RM ANOVA, Training

718 stage $* * \mathrm{p}=0.0045$. (g) Variation coefficient of the 8-lick sequence inter-lick intervals for prematurely and

719 timely initiated trials, at early and late learning stages, Two-way RM ANOVA, Training stage **p=0.0034,

720 Interaction Training stage $\mathrm{x}$ Type of trial $\mathrm{p}=0.0013$.

\section{Supplementary Figure 8. Striatal activity also marks transitions between behavioral states of the}

722 task in adolescent animals. (a) Representative raster plots and PETH of striatal units showing firing rate

723 modulations related to port entry and/or port exit. (b) Proportion of neurons showing task related firing

724 rate modulations. (c-f) Population PETH and average firing rates aligned to port entry (top panels) and to

725 port exit (bottom panels) for (c) striatal neurons showing only port entry related activity, (d) striatal

726 neurons showing only port exit related activity, (e) striatal neurons showing activity modulations at both

727 port entry and port exit and (f) striatal neurons showing higher activity during licking behavior. In (c) to

728 (f), it is represented the mean (solid lines) and SE (shaded area). Colored bars over the $\mathrm{x}$ axis show the 729 interval used to detect firing rate modulations (red: entry, orange: exit, purple: active during the task).

730 Color code for the mnFR is shown on the right (a).

\section{Supplementary Figure 9. Anticipatory, reward responsive and exit activity in striatal neurons of}

732 adolescent rats. (a) Average firing rate of entry-related neurons responding at port entry, during timely 733 and premature trials, for trials with an 8-lick sequence and trials where less than 8 licks were emitted 734 (diagram on top shows the types of trials analyzed). It is represented the mean (solid lines) and SE (shaded 735 area). Colored bars over the $\mathrm{x}$ axis show the interval used to detect firing rate modulations (-640 to -80 736 ms). (b) Average striatal activity corresponding to the PETH shown in a. (c) Population PETH and 737 Average firing rates of striatal units showing positive firing rate modulations during reward delivery, 738 reward omission and premature trials. Below, bar graphs showing statistical comparisons between trial 
739 conditions. (d) PETH showing lick rates during the three trial conditions, centered at the 8th lick, with its

740 corresponding average and statistical analysis at the bottom. (e) Discrimination index for each of the

741 groups of neurons shown in b-d, Mixed-effects model (REML) * $\mathrm{p}=0.0164$. (f) PETH showing lick rates

742 during the three trial conditions, centered at the 8th lick, with its corresponding average at the bottom.

743 Color code for the normalized activity is shown on the right. (g) Average firing rate of neurons responding

744 to port exit, during premature and timely trials, for trials with an 8-lick sequence and trials where less than

7458 licks were emitted. (h) Average striatal activity corresponding to the PETH shown in g, Two-way RM

746 ANOVA, Licks’ sequence $* * *$ p $<0.0001$.

747 Supplementary Figure 10. Localization of recording tetrodes. Schematic representation of the

748 electrodes' placement for each of the animals, based on the electrode tips visible in the histological 749 analysis, adapted from Paxinos’ Atlas (Paxinos and Watson, 2007). Animals with incorrect electrode 750 placement (gray squares) were excluded from the electrophysiological analysis but not from the 751 behavioral analysis.

\section{References}

753 Addicott, M.A., Pearson, J.M., Sweitzer, M.M., Barack, D.L., and Platt, M.L. (2017). A Primer on 754 Foraging and the Explore/Exploit Trade-Off for Psychiatry Research. Neuropsychopharmacology 42, 755 1931-1939.

756 Andrzejewski, M.E., Schochet, T.L., Feit, E.C., Harris, R., McKee, B.L., and Kelley, A.E. (2011). A 757 comparison of adult and adolescent rat behavior in operant learning, extinction, and behavioral inhibition 758 paradigms. Behav. Neurosci. 125, 93-105.

759 Asaad, W.F., Lauro, P.M., Perge, J.A., and Eskandar, E.N. (2017). Prefrontal Neurons Encode a Solution 760 to the Credit-Assignment Problem. J. Neurosci. 37, 6995-7007.

761 Atallah, H.E., McCool, A.D., Howe, M.W., and Graybiel, A.M. (2014). Neurons in the ventral striatum 762 exhibit cell-type-specific representations of outcome during learning. Neuron 82, 1145-1156. 
763 Bakhurin, K.I., Goudar, V., Shobe, J.L., Claar, L.D., Buonomano, D. V., and Masmanidis, S.C. (2017).

764 Differential Encoding of Time by Prefrontal and Striatal Network Dynamics. J. Neurosci. 37, 854-870.

765 Balleine, B.W., and Dickinson, A. (1998). Goal-directed instrumental action: contingency and incentive 766 learning and their cortical substrates. Neuropharmacology 37, 407-419.

767 Bari, A., and Robbins, T.W. (2013). Inhibition and impulsivity: behavioral and neural basis of response 768 control. Prog. Neurobiol. 108, 44-79.

769 Barnes, T.D., Kubota, Y., Hu, D., Jin, D.Z., and Graybiel, A.M. (2005). Activity of striatal neurons reflects 770 dynamic encoding and recoding of procedural memories. Nature 437, 1158-1161.

771 Baumann, A.A., and Odum, A.L. (2012). Impulsivity, risk taking, and timing. Behav. Processes 90, 408772414.

773 Belin, D., and Everitt, B.J. (2008). Cocaine seeking habits depend upon dopamine-dependent serial 774 connectivity linking the ventral with the dorsal striatum. Neuron 57, 432-441.

775 Bonner, A.C., and Borrero, J.C. (2018). Differential Reinforcement of Low Rate Schedules Reduce Severe 776 Problem Behavior. Behav. Modif. 42, 747-764.

777 Buckholtz, J.W., Treadway, M.T., Cowan, R.L., Woodward, N.D., Benning, S.D., Li, R., Ansari, M.S., 778 Baldwin, R.M., Schwartzman, A.N., Shelby, E.S., et al. (2010). Mesolimbic dopamine reward system 779 hypersensitivity in individuals with psychopathic traits. Nat. Neurosci. 13, 419-421.

780 Buhusi, C. V, and Meck, W.H. (2005). What makes us tick? Functional and neural mechanisms of interval 781 timing. Nat. Rev. Neurosci. 6, 755-765.

782 Carland, M.A., Thura, D., and Cisek, P. (2019). The Urge to Decide and Act: Implications for Brain 783 Function and Dysfunction. Neuroscientist 25, 491-511.

784 Costa, R.M. (2011). A selectionist account of de novo action learning. Curr. Opin. Neurobiol. 21, 579785586.

786 Dalley, J.W., and Robbins, T.W. (2017). Fractionating impulsivity: neuropsychiatric implications. Nat. 787 Rev. Neurosci. 18, 158-171.

788 Daw, N.D., Niv, Y., and Dayan, P. (2005). Uncertainty-based competition between prefrontal and 789 dorsolateral striatal systems for behavioral control. Nat. Neurosci. 8, 1704-1711.

790 Donnelly, N.A., Holtzman, T., Rich, P.D., Nevado-Holgado, A.J., Fernando, A.B.P., Van Dijck, G., 791 Holzhammer, T., Paul, O., Ruther, P., Paulsen, O., et al. (2014). Oscillatory activity in the medial 792 prefrontal cortex and nucleus accumbens correlates with impulsivity and reward outcome. PLoS One 9, 793 e111300.

794 Emmons, E.B., De Corte, B.J., Kim, Y., Parker, K.L., Matell, M.S., and Narayanan, N.S. (2017). Rodent 795 Medial Frontal Control of Temporal Processing in the Dorsomedial Striatum. J. Neurosci. 37, 8718-8733. 
796 Engeln, M., Ansquer, S., Dugast, E., Bezard, E., Belin, D., and Fernagut, P.-O. (2016). Multi-facetted 797 impulsivity following nigral degeneration and dopamine replacement therapy. Neuropharmacology 109, $79869-77$.

799 Ersche, K.D., Ward, L.H.E., Lim, T.-V., Lumsden, R.J., Sawiak, S.J., Robbins, T.W., and Stochl, J. (2019).

800 Impulsivity and compulsivity are differentially associated with automaticity and routine on the Creature of 801 Habit Scale. Pers. Individ. Dif. 150, 109493.

802 Evenden, J.L. (1998). The pharmacology of impulsive behaviour in rats III: the effects of amphetamine, 803 haloperidol, imipramine, chlordiazepoxide and ethanol on a paced fixed consecutive number schedule. 804 Psychopharmacology (Berl). 138, 295-304.

805 Everitt, B.J., Belin, D., Economidou, D., Pelloux, Y., Dalley, J.W., and Robbins, T.W. (2008). Review. 806 Neural mechanisms underlying the vulnerability to develop compulsive drug-seeking habits and addiction. 807 Philos. Trans. R. Soc. Lond. B. Biol. Sci. 363, 3125-3135.

808 Floresco, S.B., St Onge, J.R., Ghods-Sharifi, S., and Winstanley, C.A. (2008). Cortico-limbic-striatal 809 circuits subserving different forms of cost-benefit decision making. Cogn. Affect. Behav. Neurosci. 8, 810 375-389.

811 Geddes, C.E., Li, H., and Jin, X. (2018). Optogenetic Editing Reveals the Hierarchical Organization of 812 Learned Action Sequences. Cell 174, 32-43.e15.

813 Gibbon, J. (1977). Scalar expectancy theory and Weber’s law in animal timing. Psychol. Rev. 84, 279814325.

815 Gillan, C.M., Robbins, T.W., Sahakian, B.J., van den Heuvel, O.A., and van Wingen, G. (2016a). The role 816 of habit in compulsivity. Eur. Neuropsychopharmacol. 26, 828-840.

817 Gillan, C.M., Kosinski, M., Whelan, R., Phelps, E.A., and Daw, N.D. (2016b). Characterizing a 818 psychiatric symptom dimension related to deficits in goal-directed control. Elife 5.

819 Gouvêa, T.S., Monteiro, T., Motiwala, A., Soares, S., Machens, C., and Paton, J.J. (2015). Striatal 820 dynamics explain duration judgments. Elife 4.

821 Graybiel, A.M. (1998). The Basal Ganglia and Chunking of Action Repertoires. Neurobiol. Learn. Mem. $82270,119-136$.

823 Graybiel, A.M. (2008). Habits, rituals, and the evaluative brain. Annu Rev Neurosci 31, 359-387.

824 Green, L., Myerson, J., and Ostaszewski, P. (1999). Discounting of delayed rewards across the life span: 825 age differences in individual discounting functions. Behav. Processes 46, 89-96.

826 Gremel, C.M., and Costa, R.M. (2013). Orbitofrontal and striatal circuits dynamically encode the shift 827 between goal-directed and habitual actions. Nat. Commun. 4, 2264.

828 Hamid, A.A., Frank, M.J., and Moore, C.I. (2021). Wave-like dopamine dynamics as a mechanism for 829 spatiotemporal credit assignment. Cell 184, 2733-2749.e16. 
830 Hazan, L., Zugaro, M., and Buzsáki, G. (2006). Klusters, NeuroScope, NDManager: A free software suite 831 for neurophysiological data processing and visualization. J. Neurosci. Methods 155, 207-216.

832 Her, E.S., Huh, N., Kim, J., and Jung, M.W. (2016). Neuronal activity in dorsomedial and dorsolateral 833 striatum under the requirement for temporal credit assignment. Sci. Rep. 6, 27056.

834 Hikosaka, O., Nakahara, H., Rand, M.K., Sakai, K., Lu, X., Nakamura, K., Miyachi, S., and Doya, K. 835 (1999). Parallel neural networks for learning sequential procedures. Trends Neurosci. 22, 464-471.

836 Histed, M.H., Pasupathy, A., and Miller, E.K. (2009). Learning substrates in the primate prefrontal cortex 837 and striatum: sustained activity related to successful actions. Neuron 63, 244-253.

838 Hogarth, L., Chase, H.W., and Baess, K. (2012). Impaired goal-directed behavioural control in human 839 impulsivity. Q. J. Exp. Psychol. 65, 305-316.

840 Jin, X., and Costa, R.M. (2010). Start/stop signals emerge in nigrostriatal circuits during sequence 841 learning. Nature 466, 457-462.

842 Jin, X., Tecuapetla, F., and Costa, R.M. (2014). Basal ganglia subcircuits distinctively encode the parsing 843 and concatenation of action sequences. Nat. Neurosci. 17, 423-430.

844 Jog, M.S. (1999). Building Neural Representations of Habits. Science (80-. ). 286, 1745-1749.

845 Kamphuis, J., Baichel, S., Lancel, M., de Boer, S.F., Koolhaas, J.M., and Meerlo, P. (2017). Sleep

846 restriction in rats leads to changes in operant behaviour indicative of reduced prefrontal cortex function. J. 847 Sleep Res. 26, 5-13.

848 Kim, H., Sul, J.H., Huh, N., Lee, D., and Jung, M.W. (2009). Role of Striatum in Updating Values of 849 Chosen Actions. J. Neurosci. 29, 14701-14712.

850 Knowlton, B.J., and Patterson, T.K. (2016). Habit Formation and the Striatum. Curr. Top. Behav. 851 Neurosci. 37, 275-295.

852 Kubota, Y., Liu, J., Hu, D., DeCoteau, W.E., Eden, U.T., Smith, A.C., and Graybiel, A.M. (2009). Stable 853 encoding of task structure coexists with flexible coding of task events in sensorimotor striatum. J.

854 Neurophysiol. 102, 2142-2160.

855 Lauwereyns, J., Watanabe, K., Coe, B., and Hikosaka, O. (2002). A neural correlate of response bias in 856 monkey caudate nucleus. Nature 418, 413-417.

857 Lennox, D.B., Miltenberger, R.G., and Donnelly, D.R. (1987). Response interruption and DRL for the 858 reduction of rapid eating. J. Appl. Behav. Anal. 20, 279-284.

859 Lovic, V., Keen, D., Fletcher, P.J., and Fleming, A.S. (2011). Early-life maternal separation and social 860 isolation produce an increase in impulsive action but not impulsive choice. Behav. Neurosci. 125, 481861491.

862 Martiros, N., Burgess, A.A., and Graybiel, A.M. (2018). Inversely Active Striatal Projection Neurons and 863 Interneurons Selectively Delimit Useful Behavioral Sequences. Curr. Biol. 28, 560-573.e5. 
864 Matell, M.S., Meck, W.H., and Nicolelis, M.A.L. (2003). Interval timing and the encoding of signal 865 duration by ensembles of cortical and striatal neurons. Behav. Neurosci. 117, 760-773.

866 Mello, G.B.M., Soares, S., and Paton, J.J. (2015). A scalable population code for time in the striatum. 867 Curr. Biol. 25, 1113-1122.

868 Monterosso, J., and Ainslie, G. (1999). Beyond discounting: possible experimental models of impulse 869 control. Psychopharmacology (Berl). 146, 339-347.

870 Namboodiri, V.M., and Hussain Shuler, M.G. (2016). The hunt for the perfect discounting function and a 871 reckoning of time perception. Curr. Opin. Neurobiol. 40, 135-141.

872 Namboodiri, V.M.K., Mihalas, S., Marton, T.M., and Hussain Shuler, M.G. (2014). A general theory of 873 intertemporal decision-making and the perception of time. Front. Behav. Neurosci. 8.

874 Neill, D.B. (1976). Frontal-striatal control of behavioral inhibition in the rat. Brain Res. 105, 89-103.

875 Nonomura, S., Nishizawa, K., Sakai, Y., Kawaguchi, Y., Kato, S., Uchigashima, M., Watanabe, M., 876 Yamanaka, K., Enomoto, K., Chiken, S., et al. (2018). Monitoring and Updating of Action Selection for 877 Goal-Directed Behavior through the Striatal Direct and Indirect Pathways. Neuron 99, 1302-1314.e5.

878 Paxinos, G., and Watson, C. (2007). The Rat Brain in Stereotaxic Coordinates, Sixth Edition (Academic 879 Press).

880 Peterson, J.D., Wolf, M.E., and White, F.J. (2003). Impaired DRL 30 performance during amphetamine 881 withdrawal. Behav. Brain Res. 143, 101-108.

882 Robbins, T.W., and Costa, R.M. (2017). Habits. Curr. Biol. 27, R1200-R1206.

883 Rueda-Orozco, P.E., and Robbe, D. (2015). The striatum multiplexes contextual and kinematic 884 information to constrain motor habits execution. Nat. Neurosci. 18, 453-460.

885 Samejima, K., Ueda, Y., Doya, K., and Kimura, M. (2005). Representation of action-specific reward 886 values in the striatum. Science 310, 1337-1340.

887 Scheres, A., Dijkstra, M., Ainslie, E., Balkan, J., Reynolds, B., Sonuga-Barke, E., and Castellanos, F.X. 888 (2006). Temporal and probabilistic discounting of rewards in children and adolescents: effects of age and 889 ADHD symptoms. Neuropsychologia 44, 2092-2103.

890 Schultz, W. (2015). Neuronal Reward and Decision Signals: From Theories to Data. Physiol. Rev. 95, 891 853-951.

892 Shin, J.H., Kim, D., and Jung, M.W. (2018). Differential coding of reward and movement information in 893 the dorsomedial striatal direct and indirect pathways. Nat. Commun. 9, 404.

894 Singer, H.S. (2016). Habitual and goal-directed behaviours and Tourette syndrome. Brain 139, 312-316.

895 Smith, K.S., and Graybiel, A.M. (2013). A dual operator view of habitual behavior reflecting cortical and 896 striatal dynamics. Neuron 79, 361-374. 
897 Spear, L.P. (2000). The adolescent brain and age-related behavioral manifestations. Neurosci Biobehav 898 Rev 24, 417-463.

899 Steinberg, L., Graham, S., O’Brien, L., Woolard, J., Cauffman, E., and Banich, M. (2009). Age 900 Differences in Future Orientation and Delay Discounting. Child Dev. 80, $28-44$.

901 Thorn, C.A., Atallah, H., Howe, M., and Graybiel, A.M. (2010). Differential dynamics of activity changes 902 in dorsolateral and dorsomedial striatal loops during learning. Neuron 66, 781-795.

903 Thura, D., and Cisek, P. (2017). The Basal Ganglia Do Not Select Reach Targets but Control the Urgency 904 of Commitment. Neuron 95, 1160-1170.e5.

905 Vandaele, Y., Mahajan, N.R., Ottenheimer, D.J., Richard, J.M., Mysore, S.P., and Janak, P.H. (2019). 906 Distinct recruitment of dorsomedial and dorsolateral striatum erodes with extended training. Elife 8.

907 Vandecasteele, M., M, S., Royer, S., Belluscio, M., Berényi, A., Diba, K., Fujisawa, S., Grosmark, A., 908 Mao, D., Mizuseki, K., et al. (2012). Large-scale recording of neurons by movable silicon probes in 909 behaving rodents. J. Vis. Exp. e3568.

910 Voon, V., Derbyshire, K., Rück, C., Irvine, M.A., Worbe, Y., Enander, J., Schreiber, L.R.N., Gillan, C., 911 Fineberg, N.A., Sahakian, B.J., et al. (2015). Disorders of compulsivity: a common bias towards learning 912 habits. Mol. Psychiatry 20, 345-352.

913 Wang, A.Y., Miura, K., and Uchida, N. (2013). The dorsomedial striatum encodes net expected return, 914 critical for energizing performance vigor. Nat. Neurosci. 16, 639-647.

915 Wassum, K.M., Cely, I.C., Balleine, B.W., and Maidment, N.T. (2011). Micro-opioid receptor activation in 916 the basolateral amygdala mediates the learning of increases but not decreases in the incentive value of a 917 food reward. J. Neurosci. 31, 1591-1599.

918 Wittmann, M., and Paulus, M.P. (2008). Decision making, impulsivity and time perception. Trends Cogn. 919 Sci. 12, 7-12.

920 Wu, H., Miller, K.J., Blumenfeld, Z., Williams, N.R., Ravikumar, V.K., Lee, K.E., Kakusa, B., Sacchet, 921 M.D., Wintermark, M., Christoffel, D.J., et al. (2018). Closing the loop on impulsivity via nucleus 922 accumbens delta-band activity in mice and man. Proc. Natl. Acad. Sci. U. S. A. 115, 192-197.

923 Yamada, H., Inokawa, H., Matsumoto, N., Ueda, Y., and Kimura, M. (2011). Neuronal basis for evaluating 924 selected action in the primate striatum. Eur. J. Neurosci. 34, 489-506.

925 Yau, Y., Dadar, M., Taylor, M., Zeighami, Y., Fellows, L.K., Cisek, P., and Dagher, A. (2020). Neural 926 Correlates of Evidence and Urgency During Human Perceptual Decision-Making in Dynamically 927 Changing Conditions. Cereb. Cortex 30, 5471-5483.

928 Yin, H.H., and Knowlton, B.J. (2006). The role of the basal ganglia in habit formation. Nat Rev Neurosci $9297,464-476$.

930 Yin, H.H., Knowlton, B.J., and Balleine, B.W. (2004). Lesions of dorsolateral striatum preserve outcome 931 expectancy but disrupt habit formation in instrumental learning. Eur. J. Neurosci. 19, 181-189. 
932 Zhou, S., Masmanidis, S.C., and Buonomano, D. V. (2020). Neural Sequences as an Optimal Dynamical 933 Regime for the Readout of Time. Neuron 108, 651-658.e5.

934 Zold, C.L., and Hussain Shuler, M.G. (2015). Theta Oscillations in Visual Cortex Emerge with Experience 935 to Convey Expected Reward Time and Experienced Reward Rate. J. Neurosci. 35, 9603-9614. 936 


\section{Figure 1}

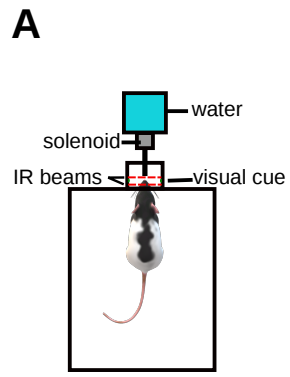

B

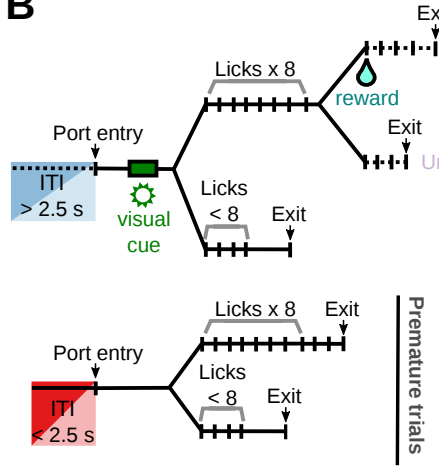

E

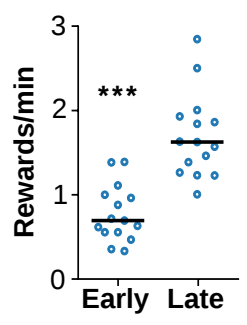

F

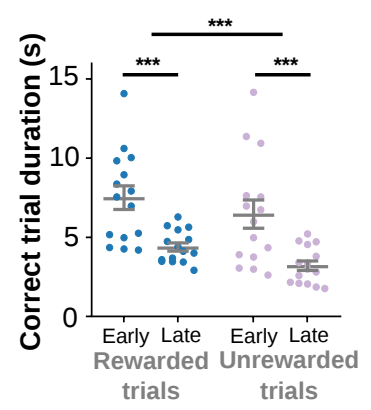

C
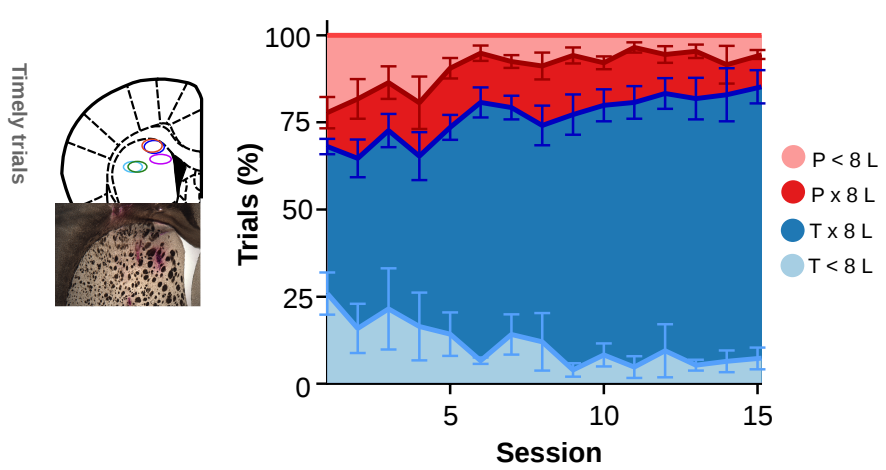

G

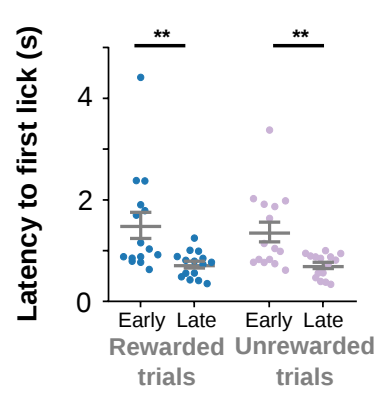

H

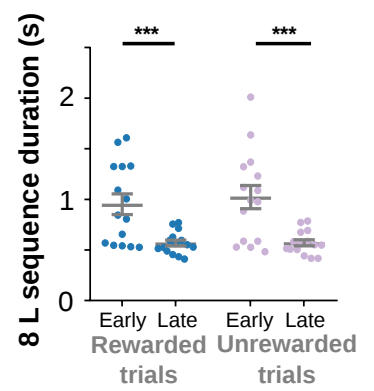




\section{Figure 2}
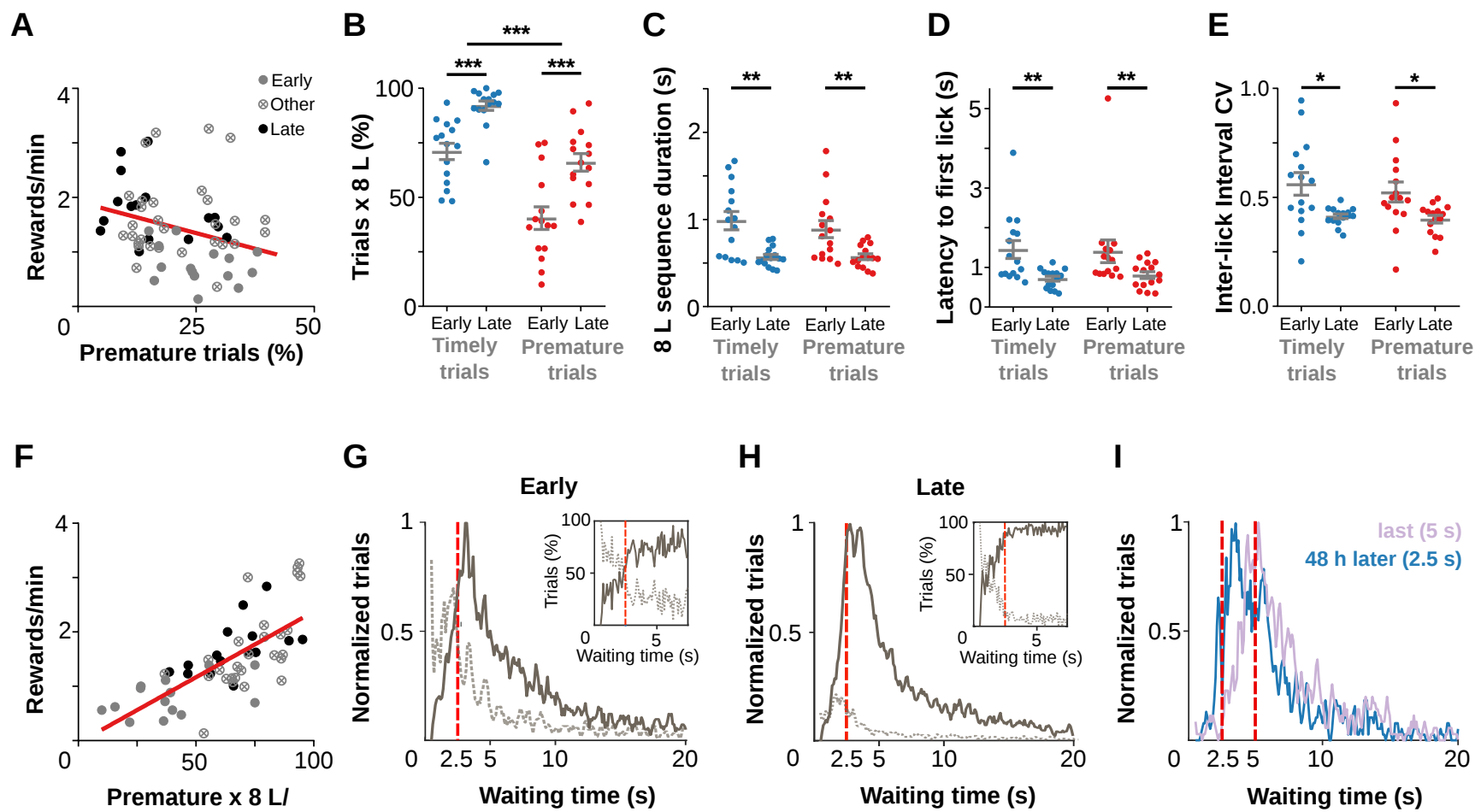

G

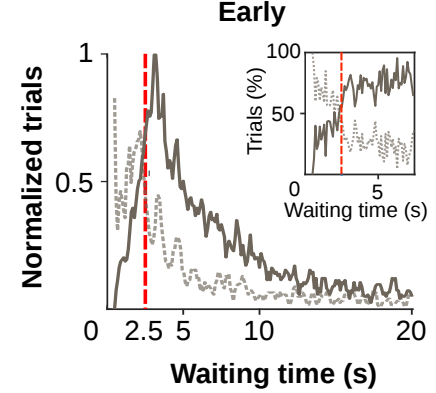

H

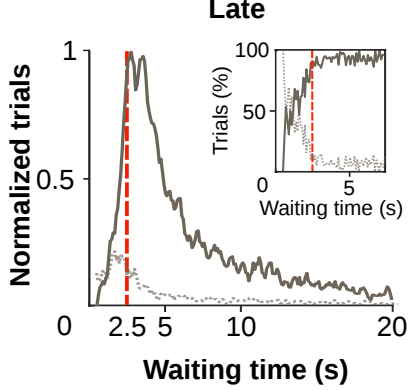

I

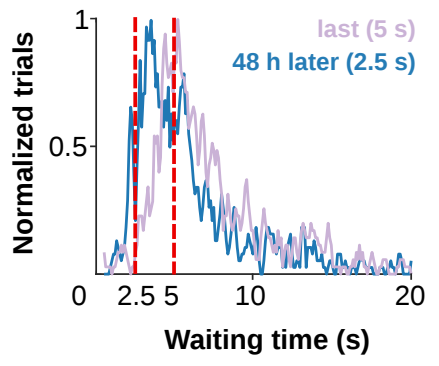

Premature trials (\%) 
bioRxiv preprint doi: https://doi.org/10.1101/2021.11.01.466347; this version posted November 3, 2021. The copyright holder for this preprint (which was not certified by peer review) is the author/funder. All rights reserved. No reuse allowed without permission.

\section{Figure 3}
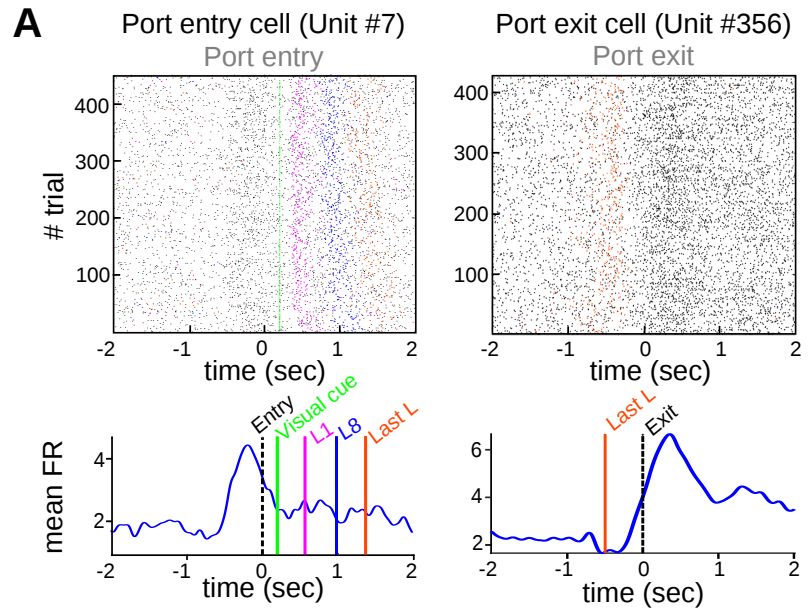

Port entry/exit cell (Unit \#285)
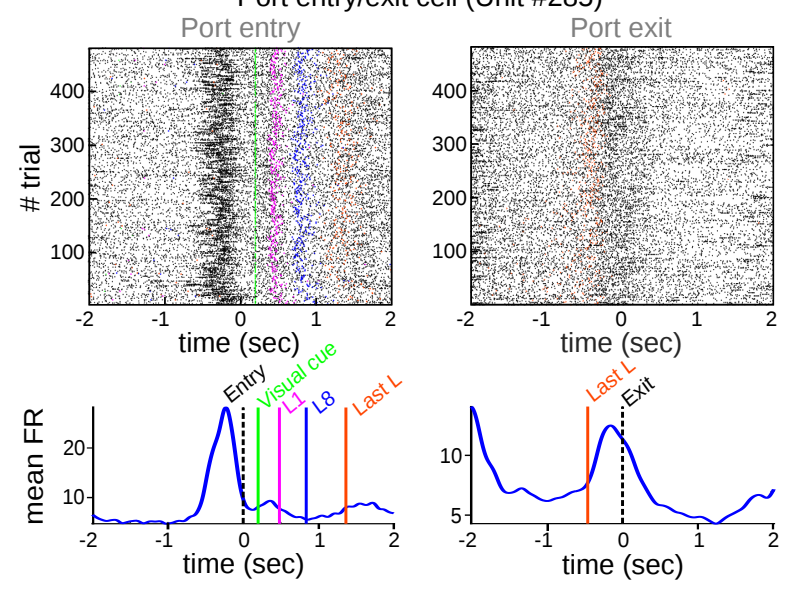

B
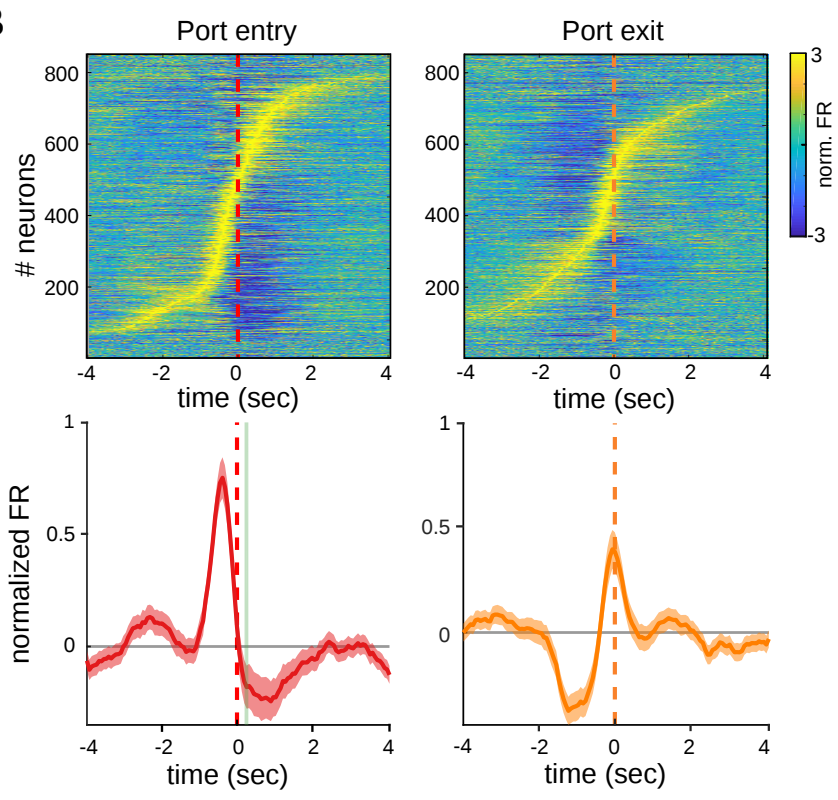

C No response

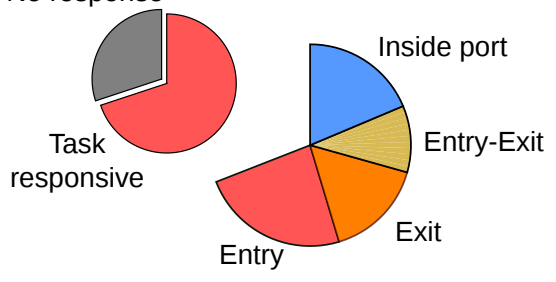

D Entry cells
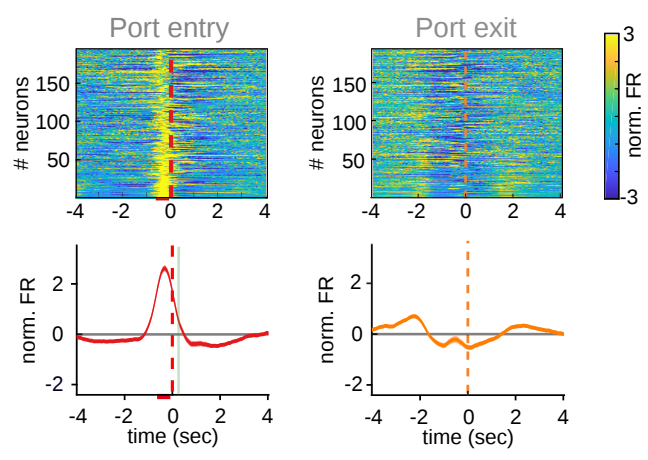

E Exit cells
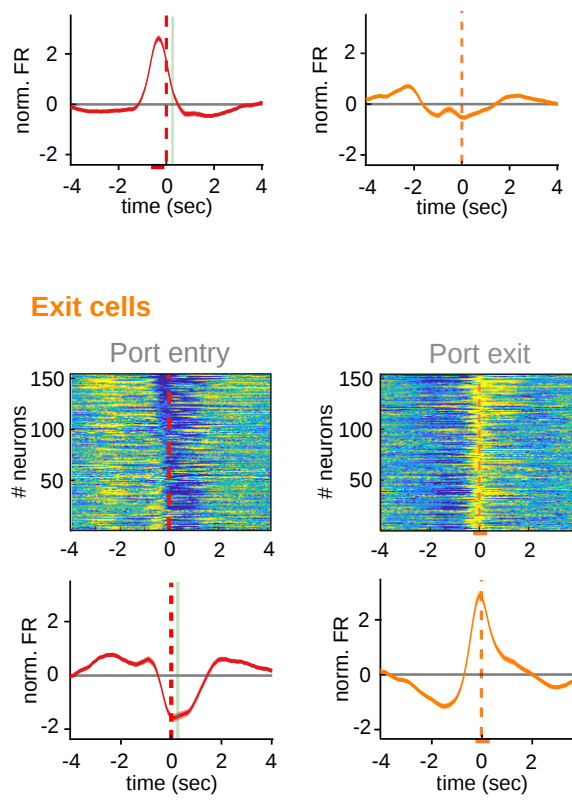

F Entry-Exit cells
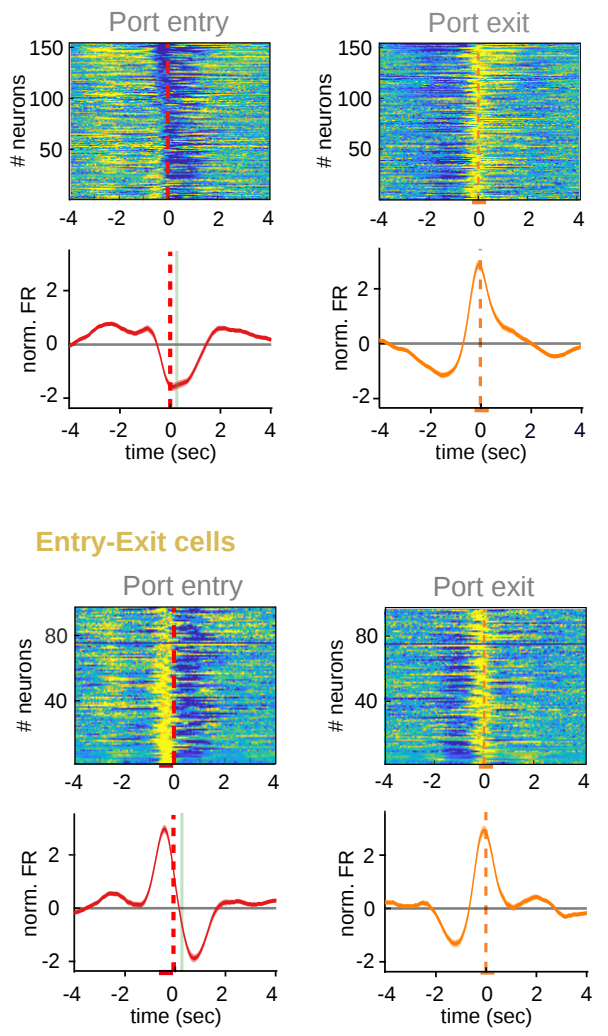

G Inside port cells
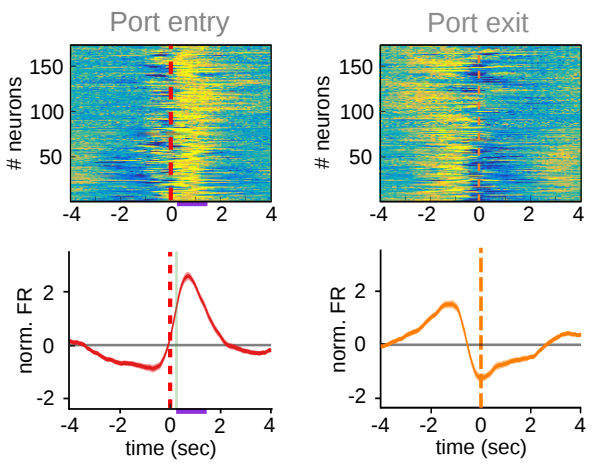


\section{Figure 4}
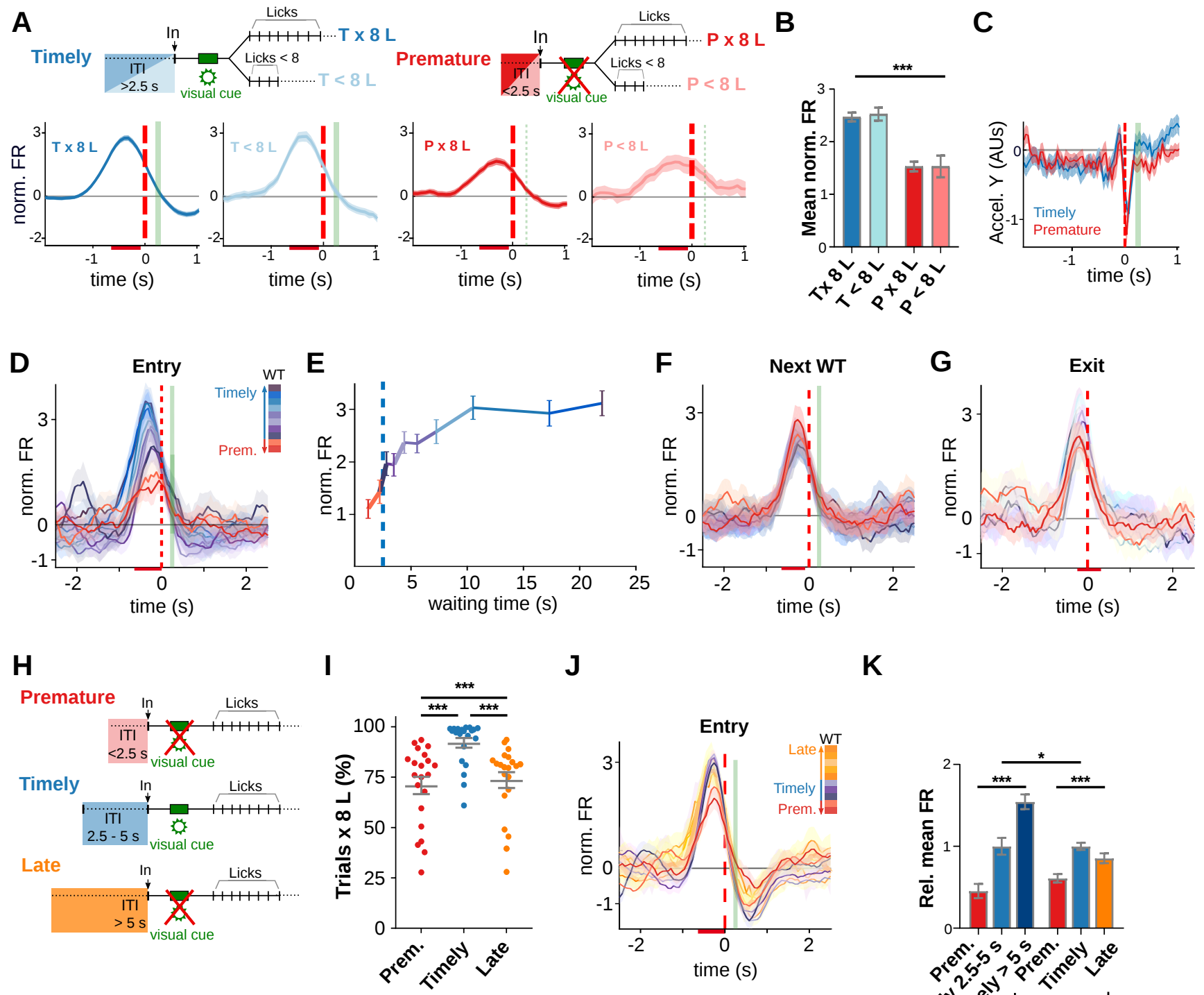

J

K
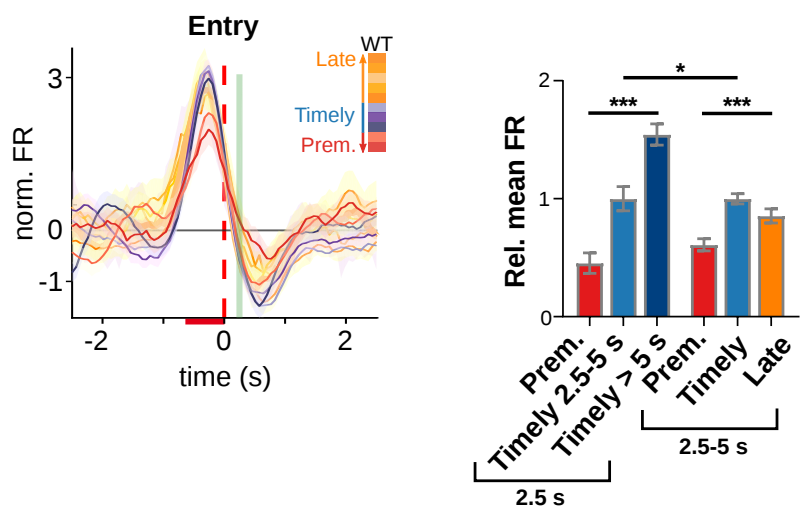
bioRxiv preprint doi: https://doi.org/10.1101/2021.11.01.466347; this version posted November 3, 2021. The copyright holder for this preprint (which was not certified by peer review) is the author/funder. All rights reserved. No reuse allowed without permission.

\section{Figure 5}

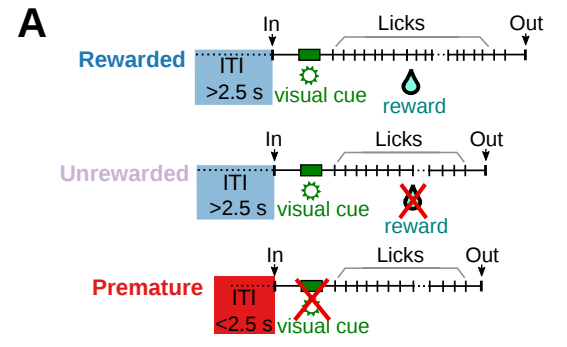

C
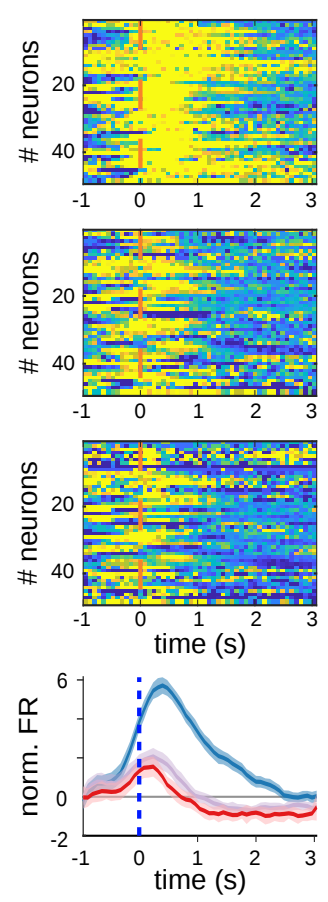

B
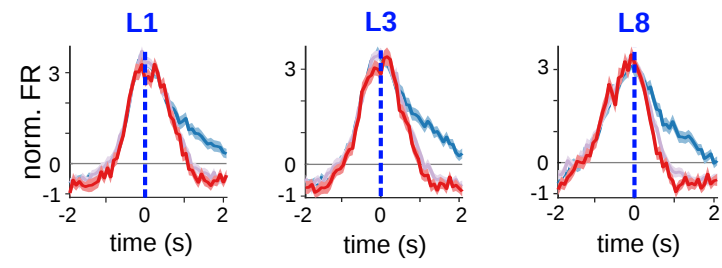

F
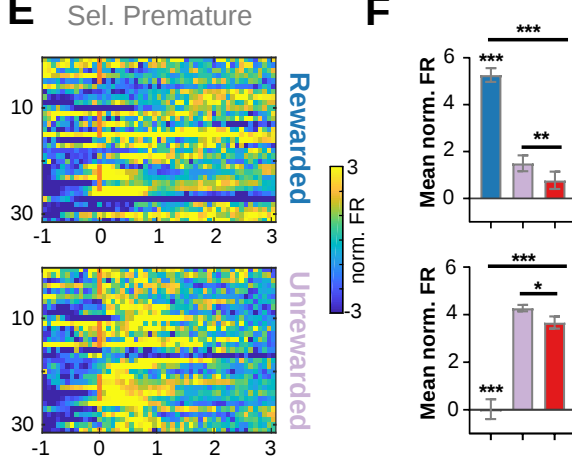

H L8
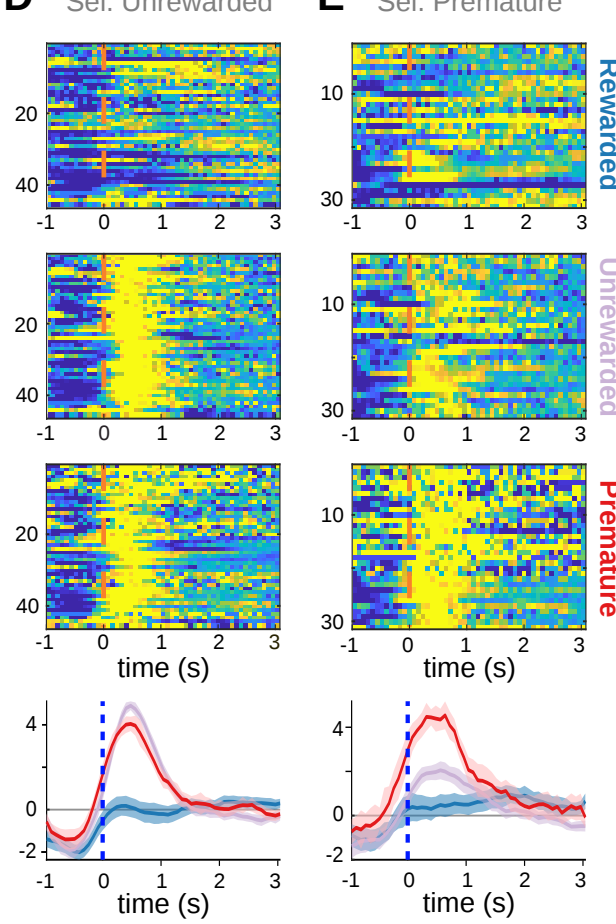
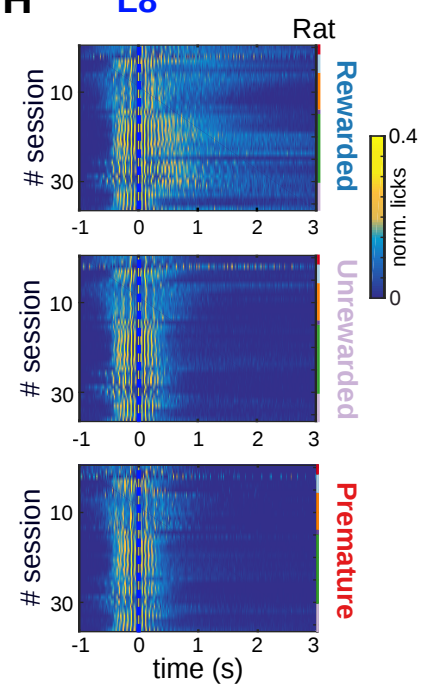

G
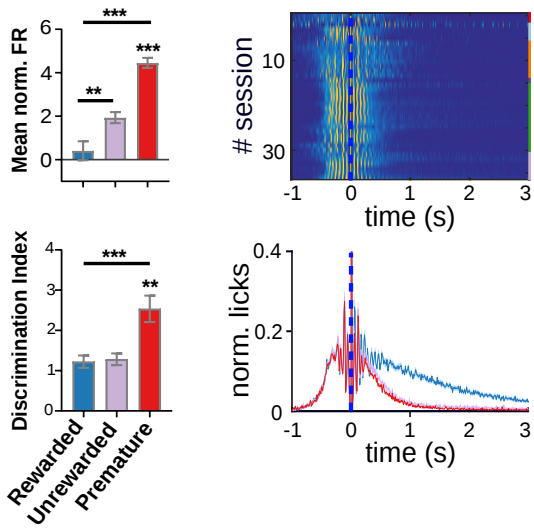
bioRxiv preprint doi: https://doi.org/10.1101/2021.11.01.466347; this version posted November 3, 2021. The copyright holder for this preprint (which was not certified by peer review) is the author/funder. All rights reserved. No reuse allowed without permission.

\section{Figure 6}
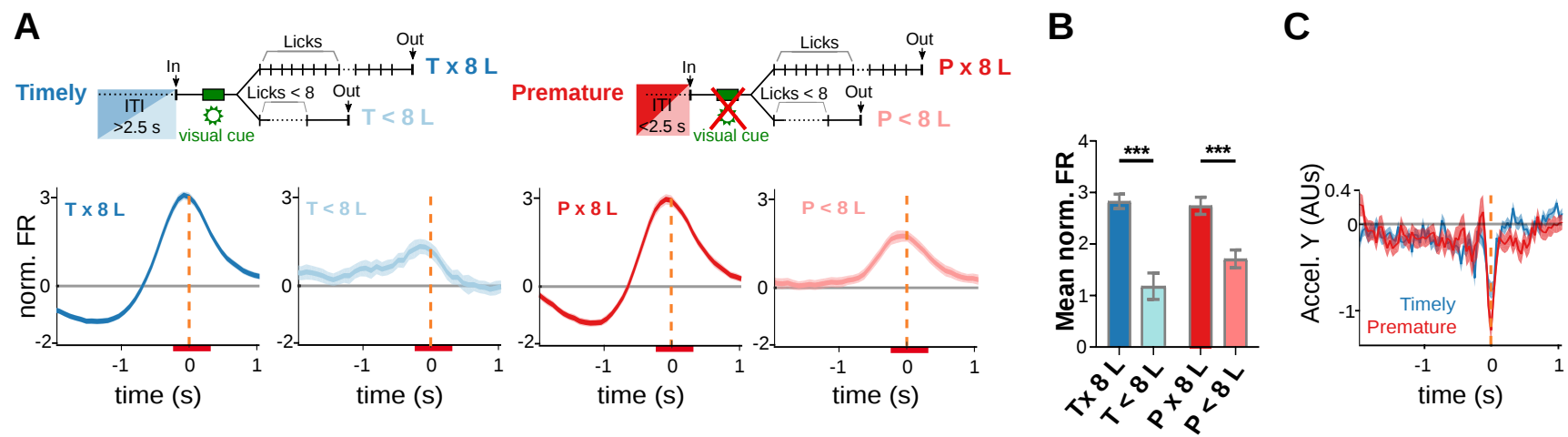


\section{Figure 7}
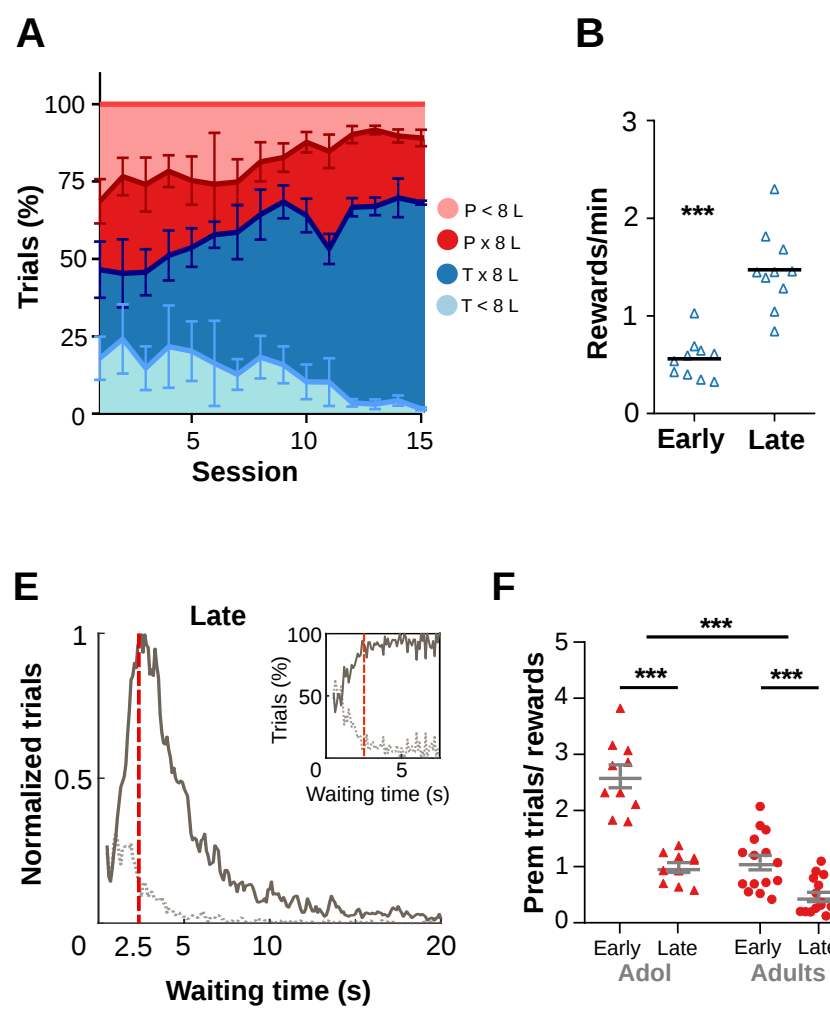

F
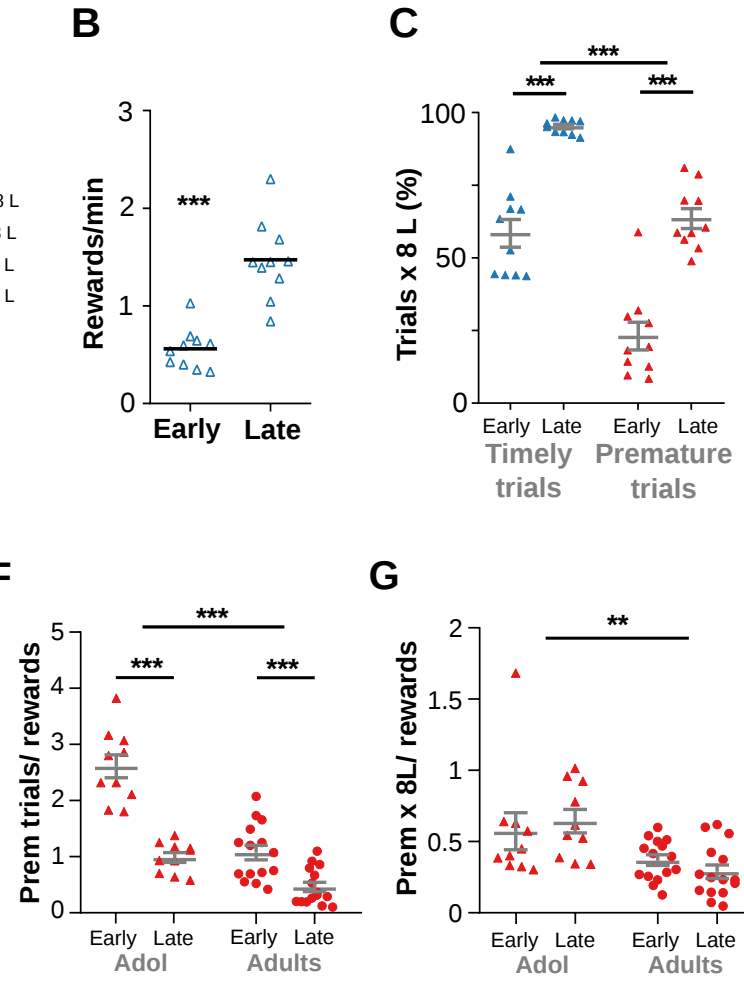

D

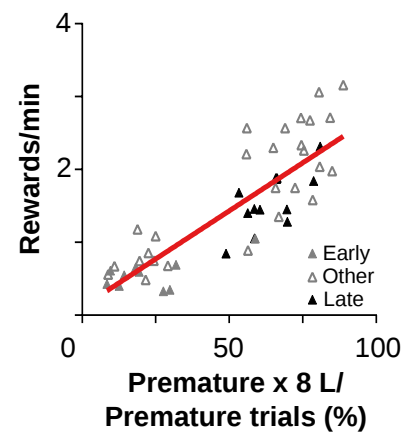

G

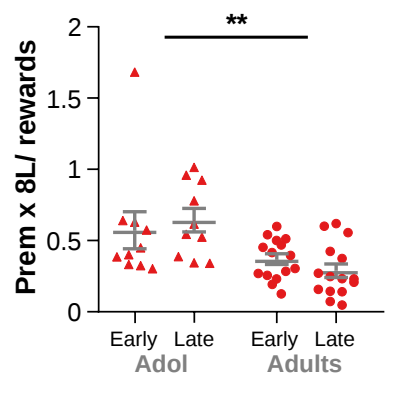

H
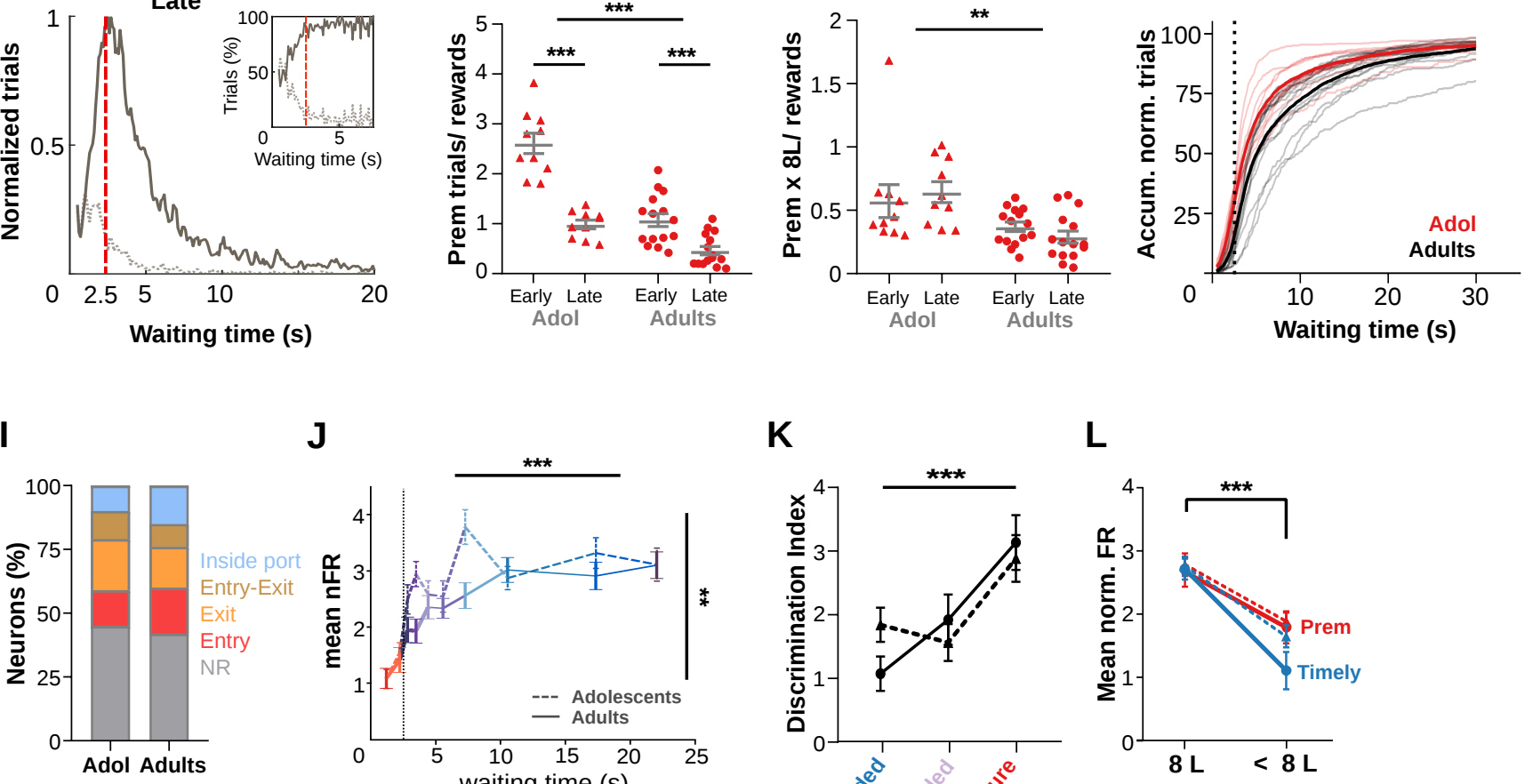

J

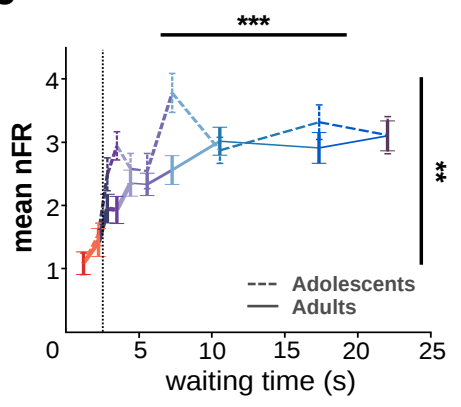

K

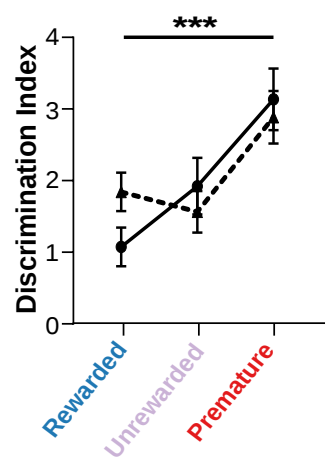

L

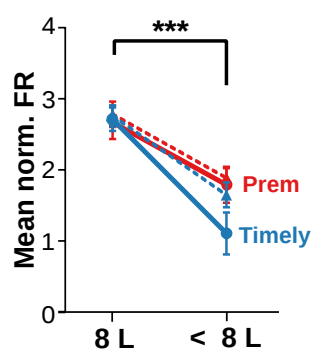




\section{Figure S1}

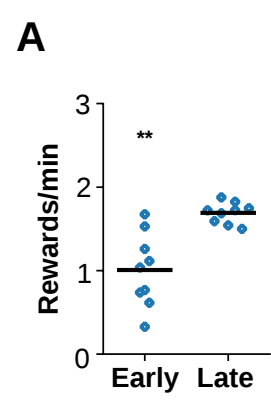

B

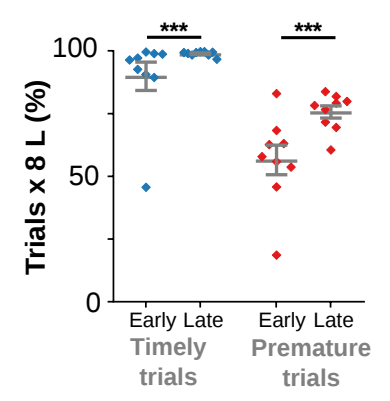

C

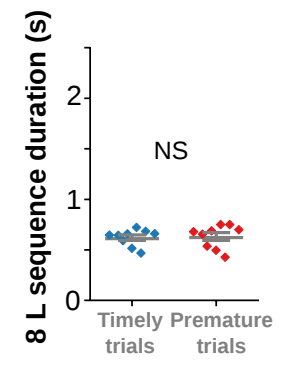

F

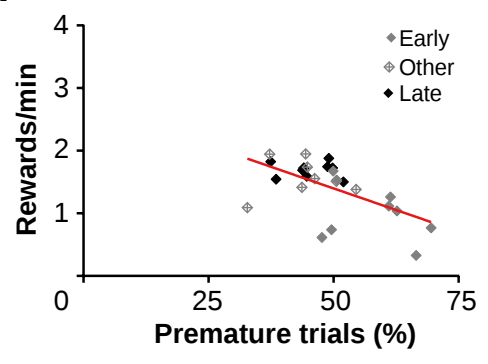

G

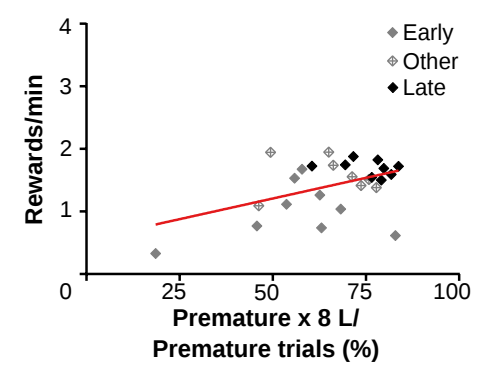

D E
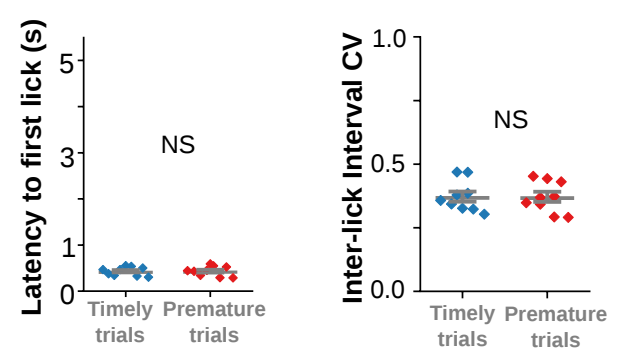

H

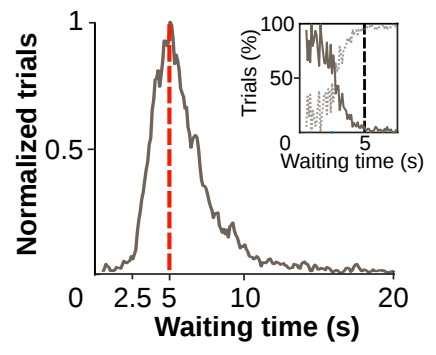


bioRxiv preprint dol: https://doi.org/10.1101/2021.11.01.466347; this version posted November 3, 2021. The copyright holder for this preprint (which was not certified by peer review) is the author/funder. All rights reserved. No reuse allowed without permission.

Figure S2

A

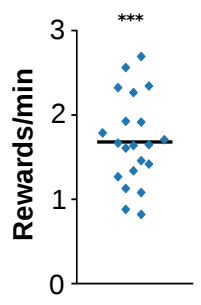

F

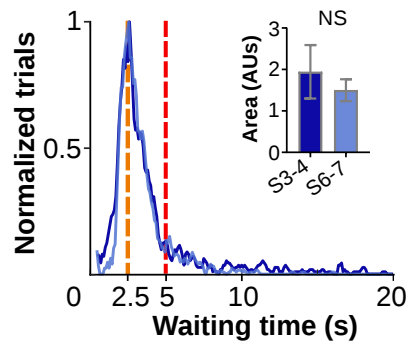

C

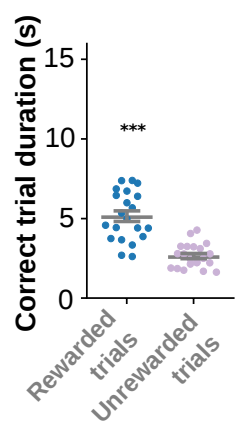

D

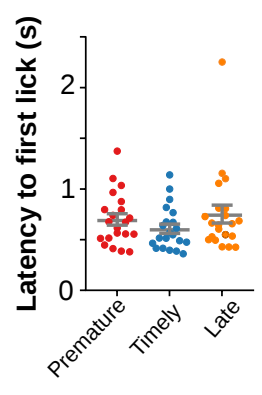

G

ITI 2.5-5 s

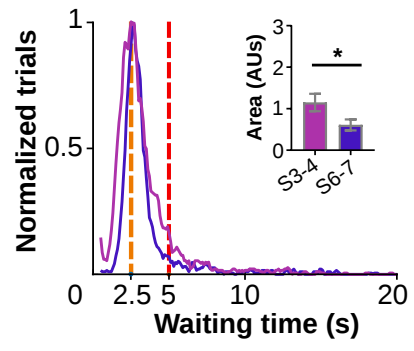

E
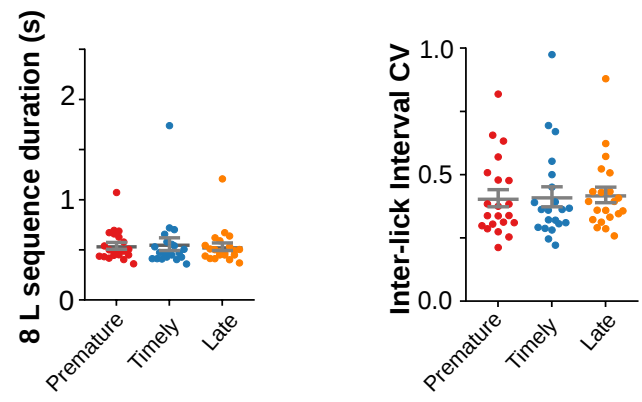
bioRxiv preprint doi: https://doi.org/10.1101/2021.11.01.466347; this version posted November 3, 2021. The copyright holder for this preprint (which was not certified by peer review) is the author/funder. All rights reserved. No reuse allowed without permission.

\section{Figure S3}
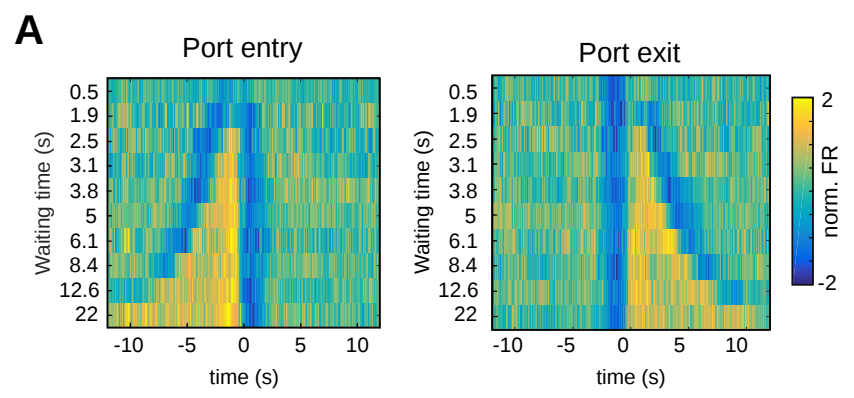

B
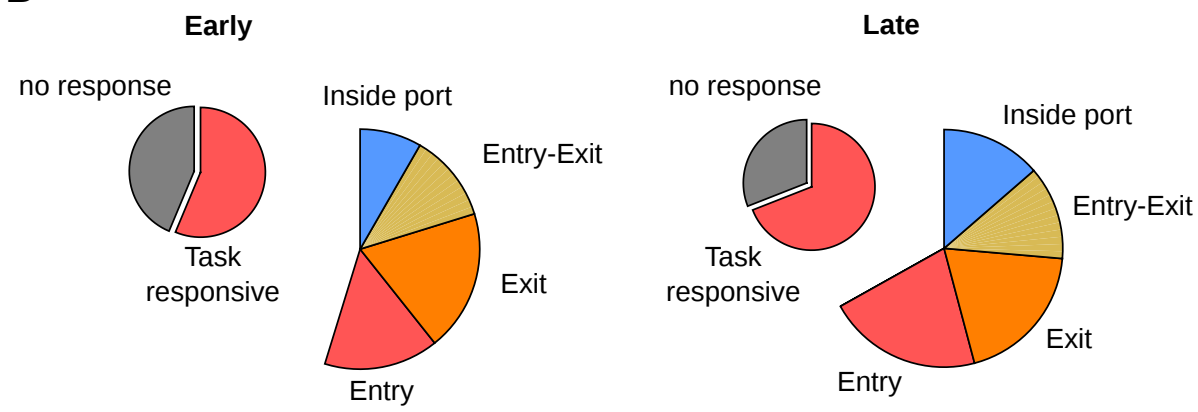


\section{Figure S4}

A Entry cells, Port entry
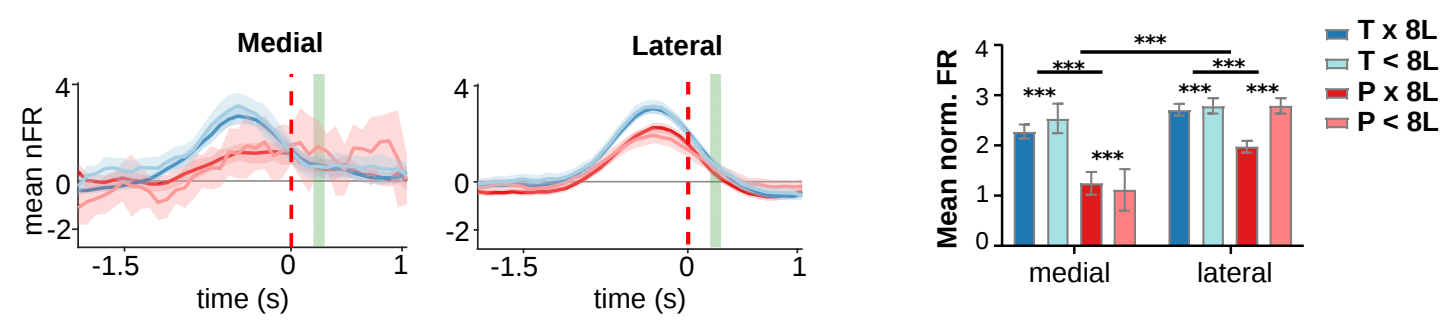

B Entry-Exit cells, Port entry
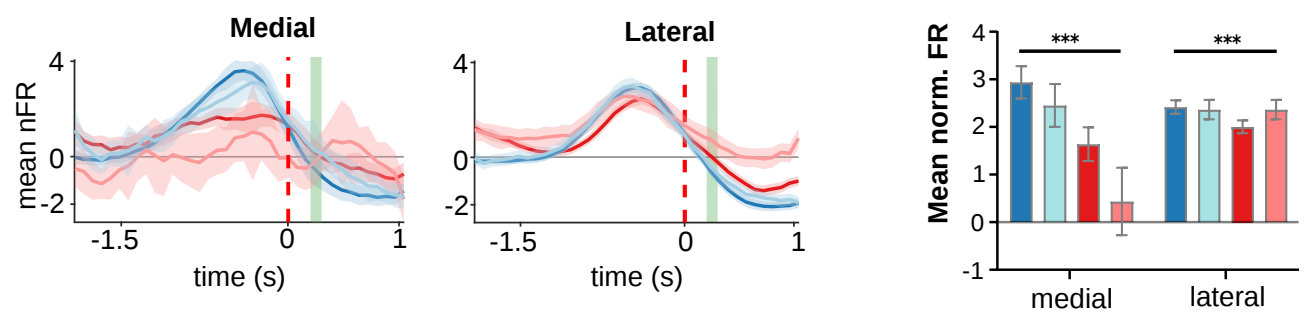

C Exit cells, Port exit
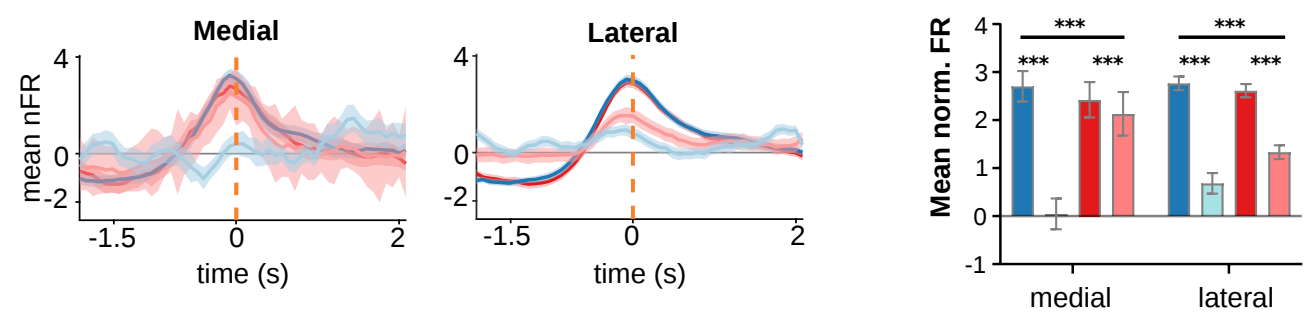

D Entry-Exit cells, Port exit
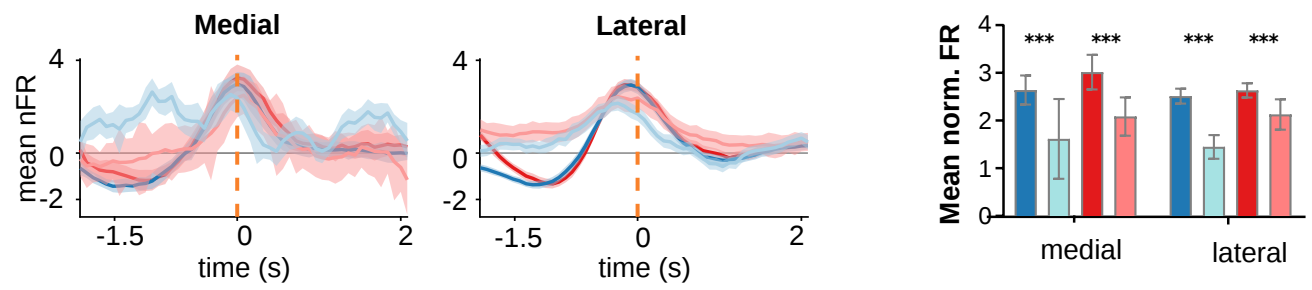
bioRxiv preprint doi: https://doi.org/10.1101/2021.11.01.466347; this version posted November 3, 2021. The copyright holder for this preprint (which was not certified by peer review) is the author/funder. All rights reserved. No reuse allowed without permission.

\section{Figure S5}

A

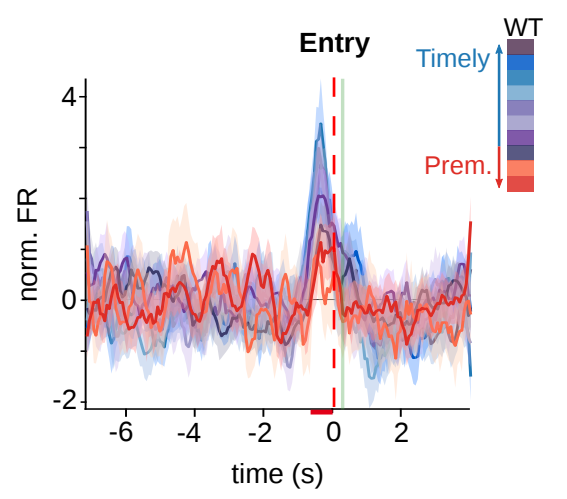

B

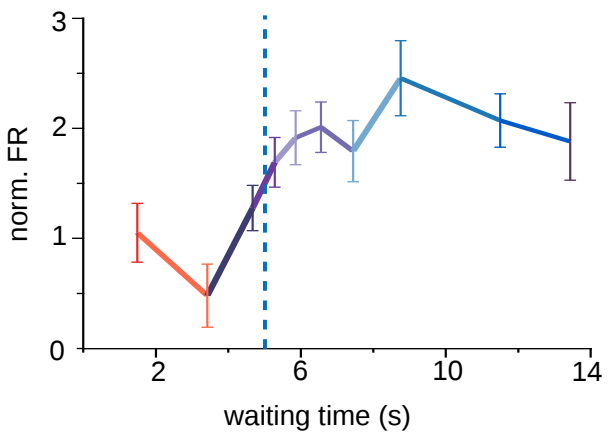


bioRxiv preprint doi: https://doi.org/10.1101/2021.11.01.466347; this version posted November 3, 2021. The copyright holder for this preprint (which was not certified by peer review) is the author/funder. All rights reserved. No reuse allowed without permission.

\section{Figure S6}
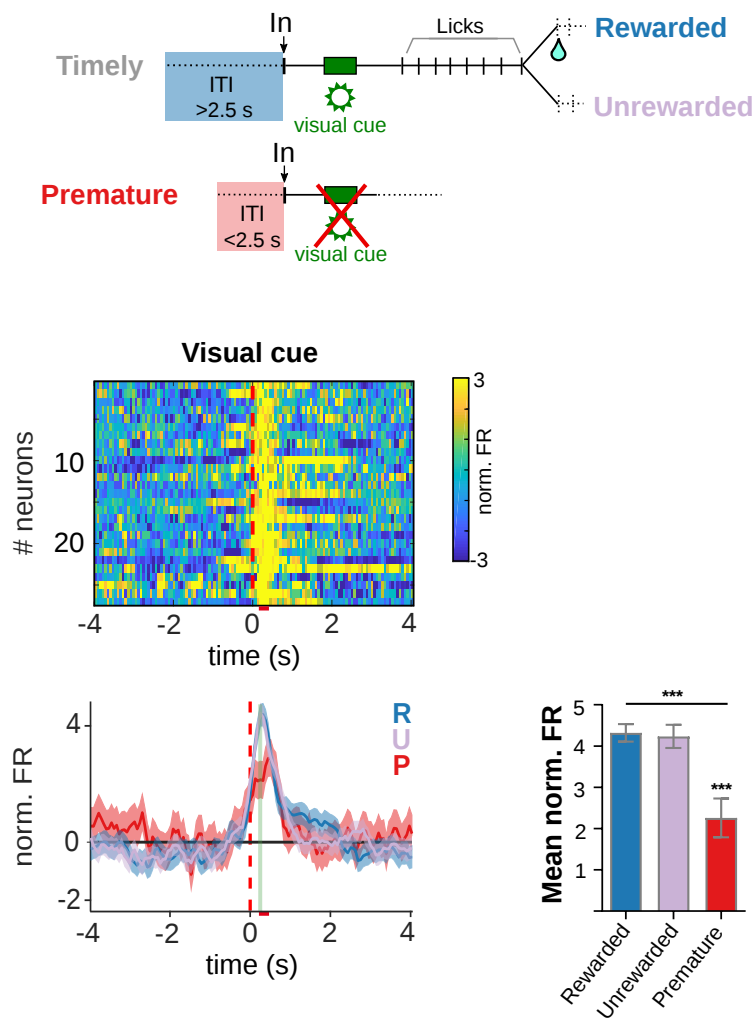


\section{Figure S7}

A

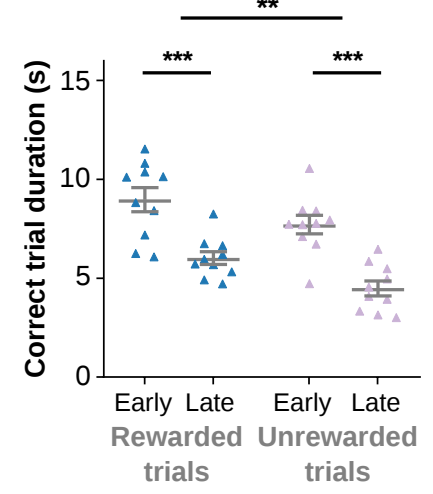

D

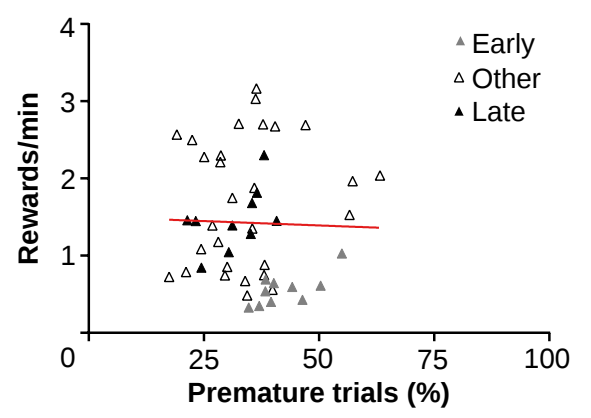

E

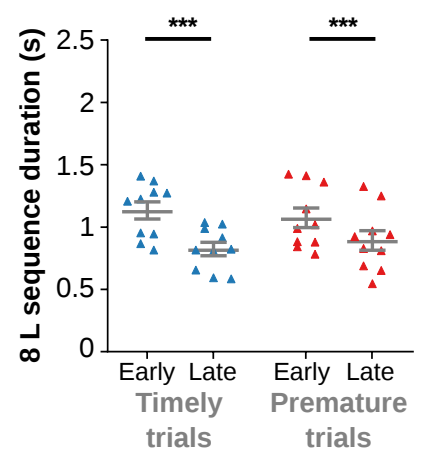

B

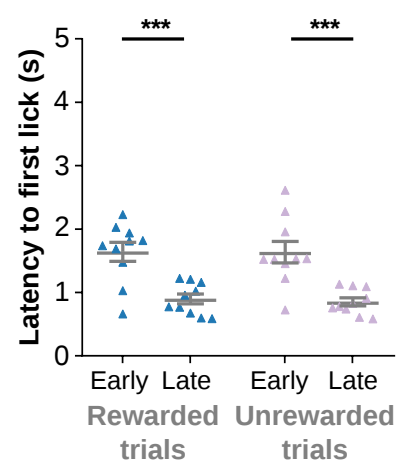

F

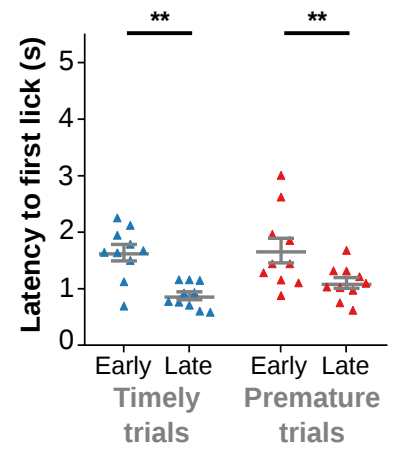

C

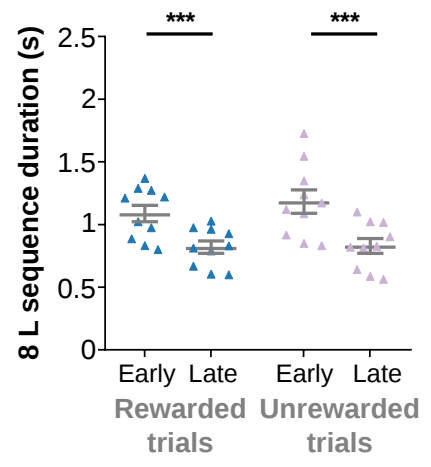

\section{G}

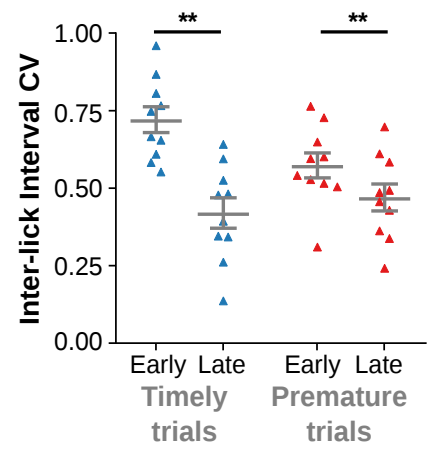


bioRxiv preprint doi: https://doi.org/10.1101/2021.11.01.466347; this version posted November 3, 2021. The copyright holder for this preprint (which was not certified by peer review) is the author/funder. All rights reserved. No reuse allowed without permission.

\section{Figure S8}

A
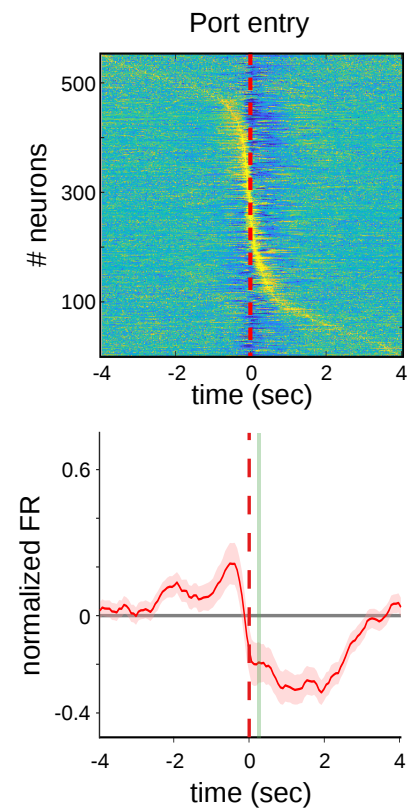

B
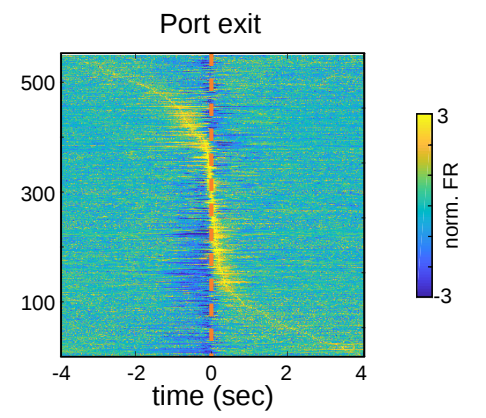

No
response

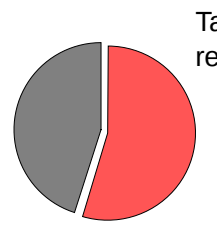

Task

responsive

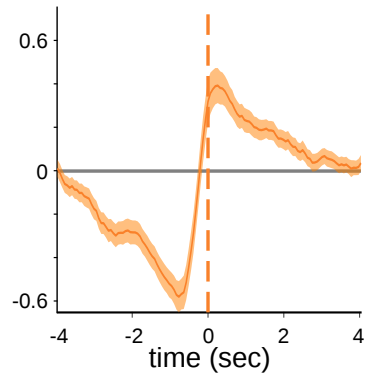

Entry

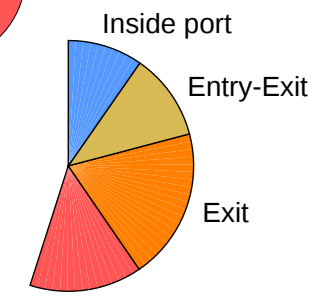

C Entry cells
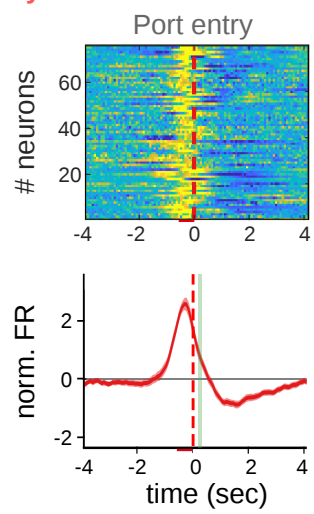

E Entry-Exit cells
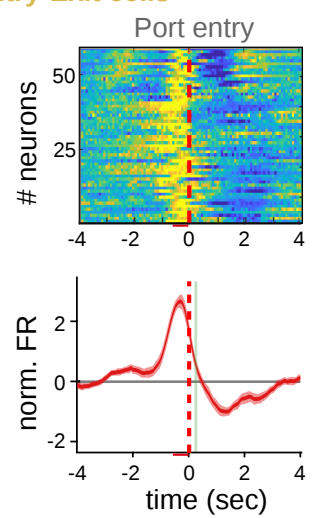

Port exit
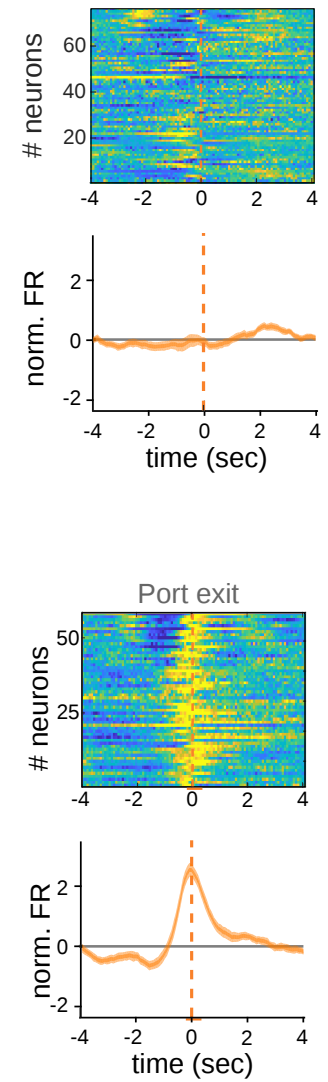

D Exit cells
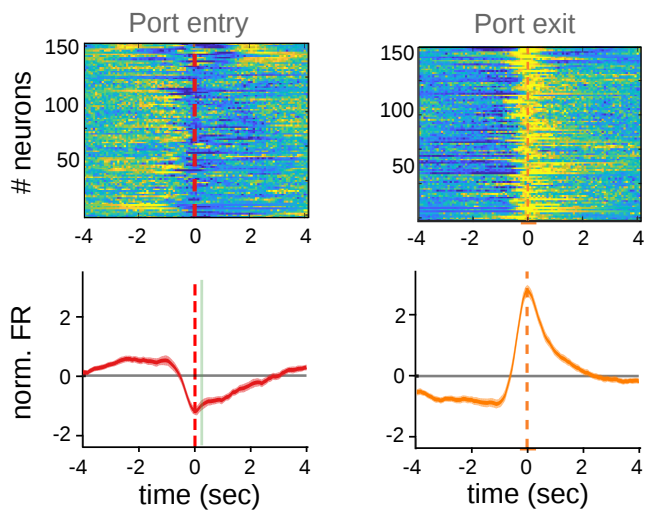

F Inside port cells
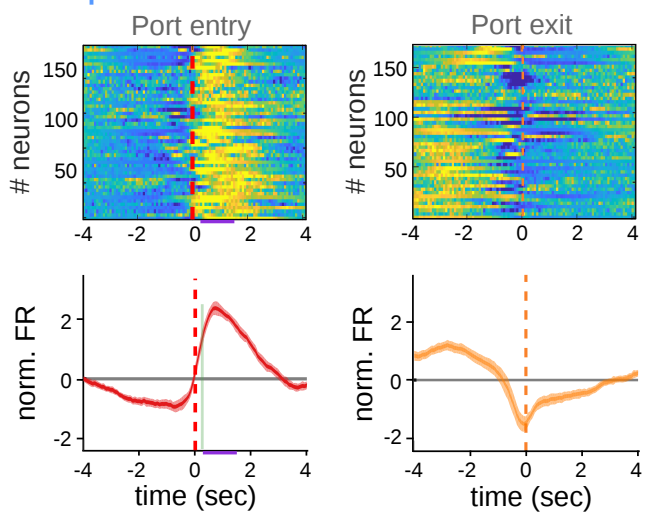
bioRxiv preprint doi: https://doi.org/10.1101/2021.11.01.466347; this version posted November 3, 2021. The copyright holder for this preprint (which was not certified by peer review) is the author/funder. All rights reserved. No reuse allowed without permission.

\section{Figure S9}

A

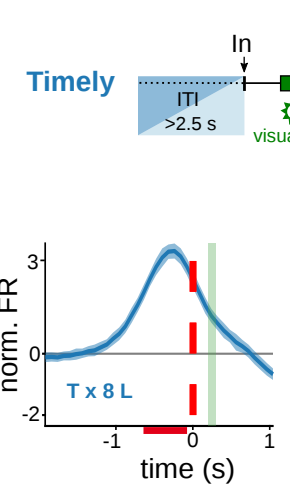

C
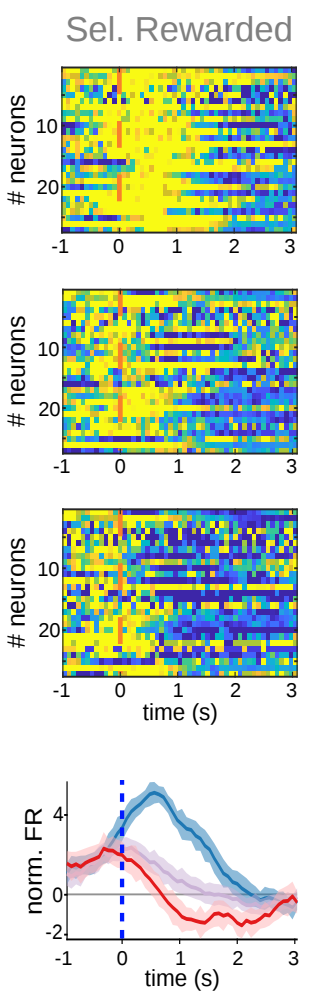

G

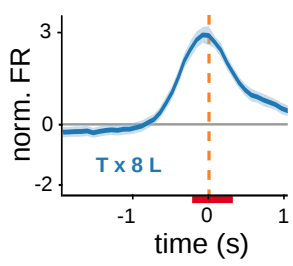

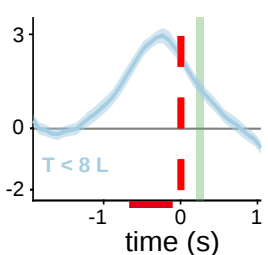

Sel. Unrewarded
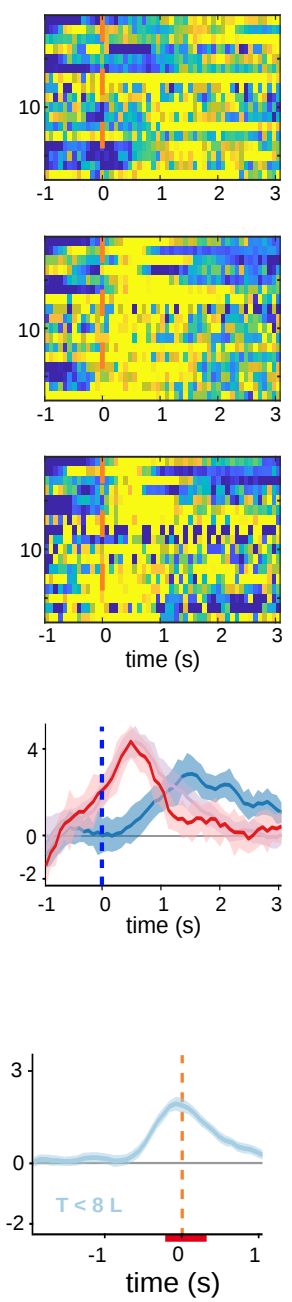
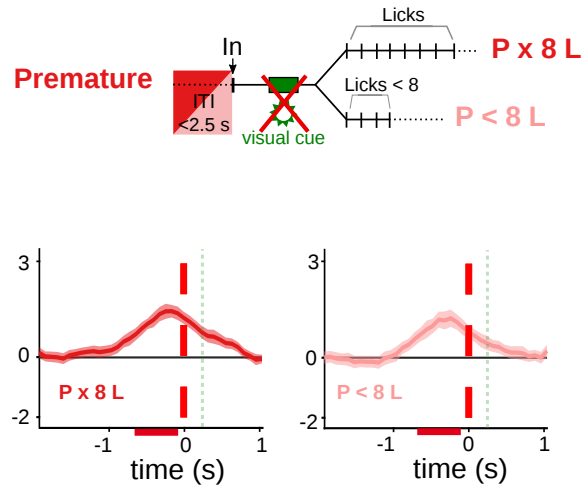

D
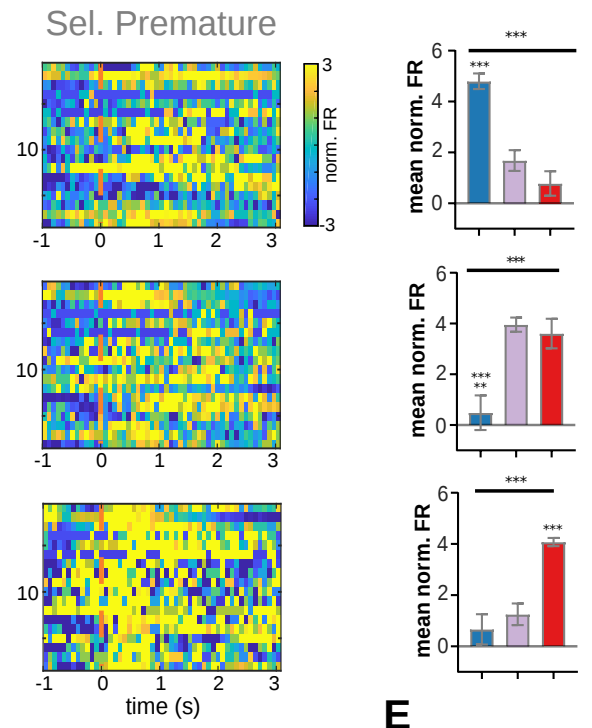

E
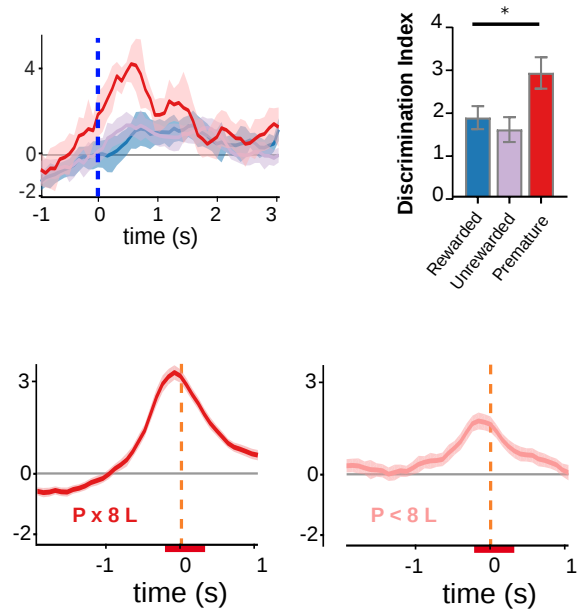

B

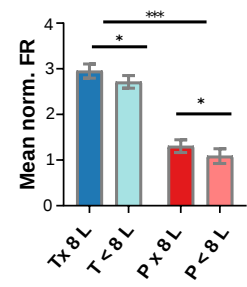

F
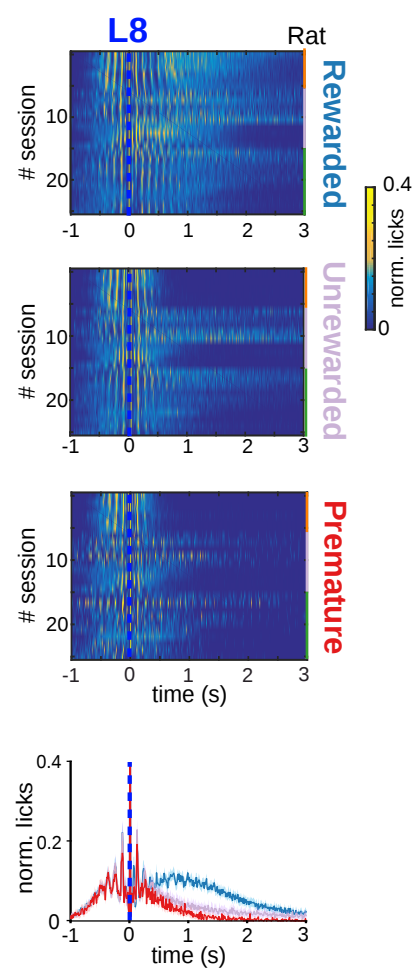

H

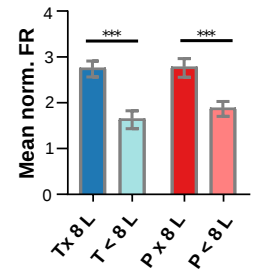


bioRxiv preprint doi: https://doi.org/10.1101/2021.11.01.466347; this version posted November 3, 2021. The copyright holder for this preprint (which was not certified by peer review) is the author/funder. All rights reserved. No reuse allowed without permission.

\section{Figure S10}

\section{Adults 2.5s WT}

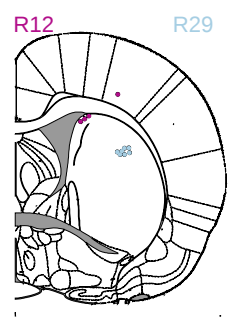

Bregma -0.12

Adults 5s WT

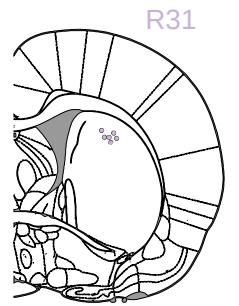

Bregma -0.12

Adults 2.5- 5s WT

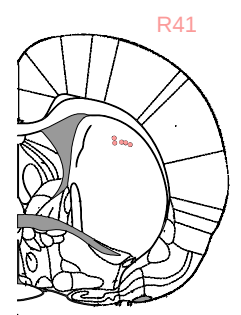

Bregma -0.12

Adolescents 2.5s WT

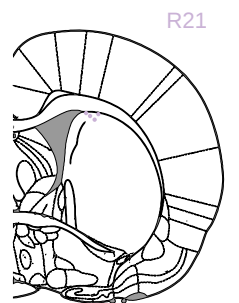

Bregma -0.12

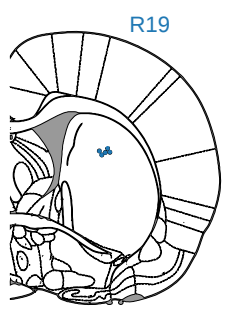

Bregma -0.12

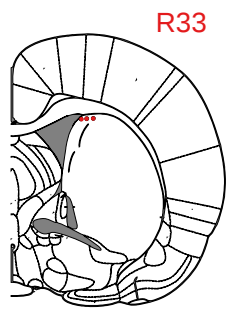

Bregma 0.00

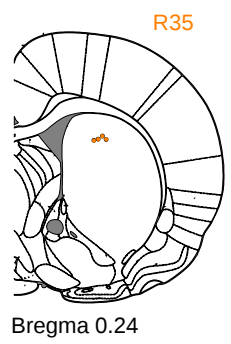

Bregma 0.24

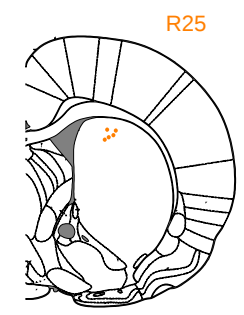

Bregma 0.24

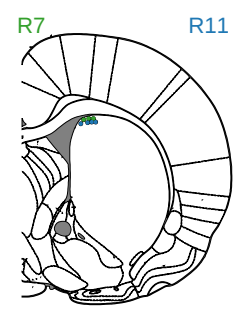

Bregma 0.24
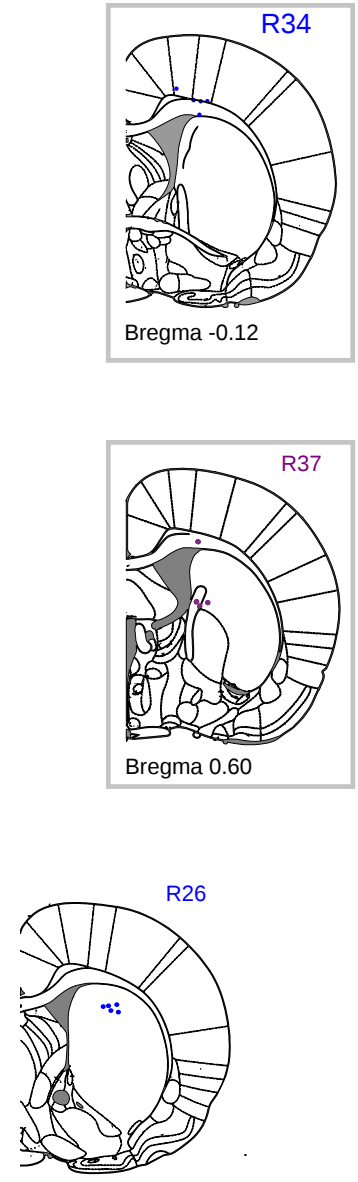

Bregma 0.24 